\title{
Conditions for Global Dynamic Stability of a Class of Resource-Bounded Model Ecosystems
}

\author{
Robert M. Seymour ${ }^{\mathrm{a}, \mathrm{b}, *}$, Gwenan Knight ${ }^{\mathrm{b}}$, Tak Fung ${ }^{\mathrm{a}, \mathrm{b}, \mathrm{c}}$ \\ ${ }^{a}$ Department of Mathematics, UCL, Gower Street, London WC1E 6BT, UK \\ ${ }^{b}$ CoMPLEX (Centre for Mathematics and Physics in the Life Sciences and Experimental \\ Biology), UCL, Gower Street, London WC1E 6BT, UK \\ ${ }^{c}$ School of Biological Sciences, Queen's University Belfast, Lisburn Road, Belfast BT9 7BL, \\ $U K$
}

Received: 12 July 2008 / Accepted: 8 February 2010 / Published online: 3 March 2010

(C) The Author(s) 2010. This article is published with open access at Springerlink.com

\begin{abstract}
This paper studies a class of dynamical systems that model multi-species ecosystems. These systems are 'resource bounded' in the sense that species compete to utilize an underlying limiting resource or substrate. This boundedness means that the relevant state space can be reduced to a simplex, with coordinates representing the proportions of substrate utilized by the various species. If the vector field is inward pointing on the boundary of the simplex, the state space is forward invariant under the system flow, a requirement that can be interpreted as the presence of non-zero exogenous recruitment. We consider conditions under which these model systems have a unique interior equilibrium that is globally asymptotically stable. The systems we consider generalize classical multispecies Lotka-Volterra systems, the behaviour of which is characterized by properties of the community (or interaction) matrix. However, the more general systems considered here are not characterized by a single matrix, but rather a family of matrices. We develop a set of 'explicit conditions' on the basis of a notion of 'uniform diagonal dominance' for such a family of matrices, that allows us to extract a set of sufficient conditions for global asymptotic stability based on properties of a single, derived matrix. Examples of these explicit conditions are discussed.
\end{abstract}

Keywords Model ecosystems · Global stability

\section{Introduction}

The study of the stability of ecosystems has a long history, both amongst ecologists and mathematical modellers. The classical view, which prevailed amongst ecologists for

\footnotetext{
*Corresponding author.

E-mail address: rms@math.ucl.ac.uk (Robert M. Seymour).
} 
a long time, was that the more complex a community is, the more stable it is (MacArthur, 1955; Elton, 1958). This intuitive view was radically challenged through studies of various mathematical models by many authors (e.g. May 1972, 1973; Gilpin, 1975; Goh and Jennings, 1977). In spite of this, doubt still remains about the relationship between complexity and stability in real communities, which may have special properties not accounted for in the 'random assemblage' mathematical models usually cited in opposition to the classical view (Begon et al., 1996, Chap. 23; Weiher and Keddy, 1999; Loreau et al., 2002; Kondoh, 2003).

Most modelling approaches to stability issues have taken a demographic perspective, concentrating on communities characterized by the number density, identity and interactions of component species (Begon et al., 1996, pp. 792-793). In particular, the most intensively explored class of models are those of Lotka-Volterra (L-V) type, defined by a system of ordinary differential equations of the form:

$$
\frac{d N_{i}}{d t}=N_{i}\left(r_{i}+\sum_{j=1}^{n} a_{i j} N_{j}\right), \quad 1 \leq i \leq n
$$

where $N_{i}$ is the population density of the $i$ th species, $r_{i}$ is its intrinsic growth rate, and $a_{i j}$ is the (constant) interaction coefficient, specifying the manner in which species $i$ interacts with species $j$ (positive, negative or zero), and the strength of this interaction. The matrix $A=\left(a_{i j}\right)$ is called the community (or interaction) matrix, and much work has been devoted to characterizing various stability properties (both local and global) in terms of properties of this matrix (reviewed in Hofbauer and Sigmund, 1998, Chap. 15; see also Siljak, 1978 and Logofet, 1993). One of the earliest and most fundamental of such results says that if $A$ is a dissipative matrix and the $\mathrm{L}-\mathrm{V}$ system admits a positive equilibrium point, then the equilibrium is both unique and globally stable (Volterra, 1931; Harrison, 1979). A matrix is dissipative (called Volterra-Lyapunov stable by Hofbauer and Sigmund, 1998, Section 15.3) if there exists a positive, diagonal matrix $Q$ such that $Q A+A^{T} Q$ is negative definite. Sufficient conditions for $A$ to be dissipative have been studied by many authors: for example, $A$ is dissipative if it is anti-symmetric (Case and Casten, 1979). Of particular interest in the present context is the demonstration by Ikeda and Siljak (1980) that $A$ is dissipative if it has a dominant diagonal (Takayama, 1985, Chap. 4).

In this paper we consider a more general class of ecosystem models than those of L-V type described above - see Section 2, Equations (4) — designed to represent a fundamental aspect of competition. Competition mechanisms can be classified into two types: exploitation and interference (Miller, 1967). Exploitation competition refers to competition arising from the joint exploitation of common limiting resources, and interference competition refers to disruption of access to necessary resources for one species by the activity of another species. For example, the famous model of MacArthur and Levins (1967) analyses an exploitation-competition community in which the interaction coefficients express the degree of resource overlap between competing species, leading to a symmetric community matrix characterized in terms of niche partitioning. They showed that this system has a unique, globally stable equilibrium. The class 
of models we consider also focus primarily on exploitation competition-that is, on ecosystems that are 'resource bounded'-but leave open whether direct species-tospecies interactions are competitive or facilitative (Abrams, 1987; Bruno et al., 2003; Day and Young, 2004).

We derive various conditions for the existence of a unique equilibrium that is globally stable. In particular, we formulate and prove a generalization of the result of Ikeda and Siljak (1980) that the community matrix of an L-V system is dissipative if it has a dominant diagonal. This generalization leads to a set of explicit conditions that imply global stability. For $n$-species systems, these explicit conditions are specified by a set-function $r:\{1,2, \ldots, n\} \rightarrow\{0,1,2, \ldots, n\}$, with each such function defining a subclass of potentially globally stable systems. Such systems are then actually globally stable if a certain matrix, defined by $r$, is an $M$-matrix (i.e., a matrix whose off-diagonal entries are nonpositive and all of whose successive principal minors are positive). We give examples for 2 -species systems in which $r(i)=0$ for each $i=1,2$ (Example 4). We also consider a specific subclass of our general class of models, which we call generalized $L$-V systems (Example 5). These reduce to systems of classical L-V form (1) when there is a stable outcome in which all the underlying limiting resources are exploited (i.e. no spare capacity). However, we consider this class of $n$-species systems when this is not a possible outcome. In this case, we show that species indices $i$ for which $r(i)=0$ determine a subsystem of semi-neutral form (i.e. having no direct inter-species interactions). We also analyse generalized L-V systems when the explicit conditions are specified by the identity function $r(i)=i$ for each $i$.

The class of resource-bounded model ecosystems we consider is described in general terms in Section 2. A crucial property that we impose on these dynamical systems is that they should be 'inward pointing' on the boundary of their state space (a simplex). This implies that no species can go extinct, a property that is guaranteed for systems with (sustained) exogenous recruitment. The condition also implies that there is no stable state in which all the underlying resources are exploited. Section 3 relates this inward-pointing condition to the theory of the index of an equilibrium and the famous Poincaré-Hopf Theorem (Milnor, 1965), from which general conditions for the existence, uniqueness and global stability of an equilibrium can be obtained (Theorem 1). This is then related to properties of the Jacobian of the dynamical system. We discuss what we call 'semineutral' systems (Section 3.2). These are systems in which there are no direct species-tospecies interactions, except possibly negative self-interactions (i.e. intra-specific competition). Thus, semi-neutral systems exhibit (almost) pure exploitative competition and are sustained by exogenous recruitment. Section 4 introduces diagonal dominance and its extension to 'uniform diagonal dominance'. As noted above, our generalization of the result of Ikeda and Siljak (1980) is then proved (Theorems 2 and 3), and associated explicit conditions derived (Sections 4.3 and 4.4). Section 5 applies the general theory of Sections 3 and 4 to systems of quadratic form, and finds conditions for these to have a unique globally stable equilibrium (Theorem 6 and Proposition 5). Section 6 gives examples of explicit conditions for uniform row- and column-diagonal dominance for quadratic systems, in particular for generalized L-V systems, as discussed above. Section 7 concludes with a summary and general discussion. Appendix A contains the more technical mathematical material. 


\section{Resource-bounded ecosystems}

\subsection{The model}

Consider a system (e.g. an ecosystem) that can sustain $n$ 'species', numbered $1,2, \ldots, n$. We assume that the system has a maximum carrying capacity $K$ in the sense that the total quantitative representation of the $n$ species cannot exceed $K$. That is, if $N_{i}$ is a quantitative measure of the representation of species $i$ in some common currency, then $0 \leq N_{i} \leq K$ and $N=\sum_{i=1}^{n} N_{i} \leq K$. A species must capture some part of the capacity $K$ in order to be represented in the system. We introduce the 'spare capacity' variable:

$$
N_{0}=K-N \text {, }
$$

so that

$$
N_{0}+N_{1}+\cdots+N_{n}=K
$$

We regard the species as competing for a common resource in the form of spare capacity, some part of which they must capture in order to increase their representation. We refer to this limiting resource as the substrate for the system.

How a species is represented depends on the nature of the system. For example, $K$ and $N_{i}$ may be measured in the common currency of biomass, density or concentration. Thus, the finite carrying capacity assumption implies that an ecosystem can support only a finite quantity of biomass, which will depend on the underlying level of primary production and the net flows of energy into and out of the system. For molecular species, the common currency is usually taken to be the concentrations of the constituent (bio-)chemical species. Alternatively, if $K$ is a fixed spatial area, and the species are all sessile organisms that grow laterally into space, then the limiting substrate is a space, and $N_{i}$ can be taken as the area occupied by species $i$. This is typical of plant-species communities.

Now assume that the ecosystem dynamics has the quadratic form:

$$
\dot{N}_{i}=\sum_{j=0}^{n} a_{i}^{j} N_{j}+\sum_{j, k=0}^{n} b_{i}^{j k} N_{j} N_{k} \quad(1 \leq i \leq n) .
$$

In order to preserve the constraint (3), we take $\dot{N}_{0}=-\dot{N}_{1}-\cdots-\dot{N}_{n}$. This implies that condition (3) is maintained along trajectories with initial conditions that satisfy (3). However, additional constraints are required to ensure that trajectories remain in the biologically meaningful positive quadrant $N_{i} \geq 0$ for $0 \leq i \leq n$. We discuss such constraints later in this section.

The constants $a_{i}^{j}$ and $b_{i}^{j k}$ define species-environment and between-species interactions respectively, and satisfy the symmetry condition $b_{i}^{j k}=b_{i}^{k j}$. In particular $a_{i}^{0}$ can be regarded as the direct rate of recruitment from the external environment of species $i$ onto available substrate (spare capacity) $N_{0}$, or induced production due to some external stimulus. With this interpretation it is natural to assume that $a_{i}^{0} \geq 0$. The coefficient $a_{i}^{j}$ for $j \geq 1$ specifies a direct contribution of species $j$ to the growth rate of species $i$ that is mediated neither by interaction with the underlying substrate $N_{0}$ nor by interactions with other species. For example, these terms could represent transition rates between life stages, size 
classes or condition states (from state $j$ to state $i$ ), such as an organism in a juvenile (pre-sexual) life stage moving to a mature (sexual) stage. Alternatively, species $j$ could produce a substance that is utilized by species $i$ to enhance (or inhibit) its growth rate. For $j=i$, a natural interpretation of $a_{i}^{i}$ is as a total mortality or emigration rate for species $i$ (i.e. the rate of transition to all other states, including death or the external environment), in which case $a_{i}^{i}<0$. Thus, if $\rho_{i}$ is the positive net death or emigration rate of species $i$, then under this interpretation we require the 'conservation' condition $\rho_{i}+\sum_{j=1}^{n} a_{j}^{i}=0$. In many of the examples considered in this paper we assume that $a_{i}^{j}=0$ for $j \neq i$, so that $\rho_{i}=-a_{i}^{i}$. However, this assumption is not necessary for the general theory.

The species-substrate interaction coefficient, $2 b_{i}^{j 0}$, can be regarded as the rate of recruitment of species $i$ into the system due to the interaction of species $j$ with the underlying substrate; e.g. by production of propagules of species $i$, or by "preparing the ground" by rendering the available substrate more (or less) favourable to external recruitment of species $i$ (effectively boosting or inhibiting $a_{i}^{0}$ ). This could be negative if the action of species $j$ inhibits access of species $i$ to substrate, say by producing an inhibitory substance (i.e. interference competition). In particular, for $j=i, 2 b_{i}^{i 0}$ can be regarded as the rate at which established species $i$ utilizes available substrate to enhance its own recruitment/growth if it is positive, or as a density-dependent inhibition effect on the intrinsic recruitment rate $a_{i}^{0}$ if it is negative. On the other hand, $b_{i}^{j k}$ for $j, k \geq 1$ determines the rate of recruitment/growth (or loss) of species $i$ due to direct interaction between species $j$ and $k$ : for example, if $j$ and $k$ interact to produce a substance that promotes (or inhibits) the growth of species $i$. In particular, if $b_{i}^{i j}<0$ and $b_{j}^{i j}>0$, then $b_{i}^{i j}$ can represent the rate of loss of species $i$ due to predation by species $j$, with $b_{j}^{i j}$ the rate of gain of species $j$ due to predation on species $i$. If $b_{i}^{i j}<0$ and $b_{j}^{i j}<0$, then the interaction between species $i$ and species $j$ is one of direct interference competition, and if $b_{i}^{i j}>0$ and $b_{j}^{i j}>0$, then the interaction is mutualistic. Finally, it is perhaps most natural to suppose that $b_{i}^{00}=0$, unless the "substrate" interacts with itself autocatalytically to either promote or inhibit recruitment of species $i$. However, in general we shall not assume that $b_{i}^{00}=0$ in the formal considerations that follow.

\subsection{Proportion variables}

It is convenient to work with proportion variables, $x_{i}=N_{i} / K$ for $0 \leq i \leq n$. Thus, from (3) we have:

$$
x_{0}+x_{1}+\cdots+x_{n}=1 \text {. }
$$

Since $K$ is constant, the ecosystem dynamics (4) can be expressed in terms of the proportion variables:

$$
\dot{x}_{i}=\sum_{j=0}^{n} a_{i}^{j} x_{j}+\sum_{j, k=0}^{n} \gamma_{i}^{j k} x_{j} x_{k} \quad(1 \leq i \leq n),
$$

where $\gamma_{i}^{j k}=K b_{i}^{j k}$, and $\dot{x}_{0}=-\dot{x}_{1}-\cdots-\dot{x}_{n}$. It follows that the constraint (5) is maintained along trajectories. If the constraints $x_{i} \geq 0$ for $0 \leq i \leq n$ are also maintained along 
trajectories, then the biologically relevant state space for this dynamical system is the $n$-dimensional simplex

$$
\Delta=\left\{x=\left(x_{1}, \ldots, x_{n}\right) \in \mathbf{R}^{n}: 0 \leq x_{i} \leq \sum_{j=1}^{n} x_{j} \leq 1\right\} .
$$

Thus, we may write (6) in the form $\dot{x}=g(x)$, where $g: \Delta \rightarrow \mathbf{R}^{n}$ defines a smooth vector field on $\Delta$.

By substituting $x_{0}=1-\sum_{i=1}^{n} x_{i}$ into (6), we may write

$$
g_{i}(x)=c_{i}+\sum_{j=1}^{n} A_{i}^{j} x_{j}+\sum_{j, k=1}^{n} \Gamma_{i}^{j k} x_{j} x_{k} \quad(1 \leq i \leq n),
$$

where:

$$
\begin{aligned}
& c_{i}=a_{i}^{0}+\gamma_{i}^{00}, \\
& A_{i}^{j}=a_{i}^{j}-a_{i}^{0}+2 \gamma_{i}^{0 j}-2 \gamma_{i}^{00}, \\
& \Gamma_{i}^{j k}=\gamma_{i}^{j k}-\left(\gamma_{i}^{j 0}+\gamma_{i}^{0 k}\right)+\gamma_{i}^{00} .
\end{aligned}
$$

Here $c_{i}$ is the net rate of recruitment from or loss to the external environment; $A_{i}^{j}$ is the net direct effect of species $j$ on the growth rate of species $i$; and $\Gamma_{i}^{j k}$ is the net effect on the growth rate of species $i$ due to interactions between species $j$ and $k$ (and the substrate). Thus, $c=\left(c_{1}, \ldots, c_{n}\right)$ is an $n$-vector, $A=\left(A_{i}^{j}\right)$ is an $n \times n$ matrix, and $\Gamma_{i}=\left(\Gamma_{i}^{j k}\right)$ is a symmetric $n \times n$ matrix for each $i$, so that $\Gamma=\left(\Gamma_{1}, \ldots, \Gamma_{n}\right)$ is a $(2,1)$-tensor. We may therefore write the vector field $g: \Delta \rightarrow \mathbf{R}^{n}$ in the form

$$
g(x)=c+A x+x \cdot \Gamma x \quad(x \in \Delta) .
$$

\subsection{The inward-pointing condition}

To ensure that the constraints (5) and $x_{i} \geq 0$ for $0 \leq i \leq n$ are maintained along trajectories of the system (8), we assume that $g$ is inward pointing on the simplex boundary $\partial \Delta .{ }^{1}$ That is, if $e_{i}$ is the $i$ th unit standard coordinate vector in $\mathbf{R}^{n}$, then $g_{i}(x)=e_{i} \cdot g(x)>0$ whenever $x_{i}=0(1 \leq i \leq n)$, and $g_{0}(x)=-e \cdot g(x)>0$ whenever $x_{0}=0$ (where $\left.e=\sum_{i=1}^{n} e_{i} \in \mathbf{R}^{n}\right)$. This means that $\Delta$ is forward invariant under the flow of the dynamical system $\dot{x}=g(x)$ on $\mathbf{R}^{n}$. Because the inequalities on the boundary are strict, this also implies that no species, once established, can become extinct. In particular $g(x)$ is nowhere zero on $\partial \Delta$. This inward-pointing condition provides an important contribution to the self-sustaining nature of globally stable ecosystems.

\footnotetext{
${ }^{1}$ This condition can be weakened in most of what follows. For $\varepsilon>0$, let $U_{\varepsilon}=\{x \in \Delta: d(x, \partial \Delta)<\varepsilon\}$ be the open $\varepsilon$-neighbourhood of $\partial \Delta$ in $\Delta$. Assume there is an $\varepsilon_{0}>0$, such that, for each $0<\varepsilon<\varepsilon_{0}$, there is a convex open set $M_{\varepsilon}$ in $\Delta$ with closure $\bar{M}_{\varepsilon} \subset$ int $\Delta$ and boundary a smooth closed manifold, $\partial M_{\varepsilon} \subset U_{\varepsilon}$, with $g$ inward pointing on $\partial M_{\varepsilon}$. This implies that any arbitrarily small, non-zero representation of species $i$ will grow; i.e. once established, species $i$ cannot die out. This is similar to the permanence condition discussed by Hofbauer and Sigmund (1998, Section 12.2). However, for the sake of simplicity we shall not consider this more general situation explicitly in this paper.
} 
Example 1 (1-species systems). For the case of one species, $n=1$, we have:

$$
g_{1}\left(x_{0}, x_{1}\right)=a_{1}^{0} x_{0}+a_{1}^{1} x_{1}+\gamma_{1}^{00} x_{0}^{2}+2 \gamma_{1}^{01} x_{0} x_{1}+\gamma_{1}^{11} x_{1}^{2},
$$

with $x_{0}+x_{1}=1$. In the form (10) this is:

$$
g_{1}\left(x_{1}\right)=\left(a_{1}^{0}+\gamma_{1}^{00}\right)+\left(a_{1}^{1}-a_{1}^{0}+2 \gamma_{1}^{01}-2 \gamma_{1}^{00}\right) x_{1}+\left(\gamma_{1}^{11}-2 \gamma_{1}^{01}+\gamma_{1}^{00}\right) x_{1}^{2} .
$$

To be inward pointing on the interval $0 \leq x_{1} \leq 1$, we require $g_{1}(1,0)>0$ and $g_{1}(0,1)<0$. That is,

$$
g_{1}(1,0)=a_{1}^{0}+\gamma_{1}^{00}>0 \quad \text { and } \quad g_{1}(0,1)=a_{1}^{1}+\gamma_{1}^{11}<0 .
$$

For example, taking $\gamma_{1}^{00}=0$ (no interaction of the substrate with itself that affects the growth of species 1 ), we then require the exogenous recruitment rate to available substrate $a_{1}^{0}>0$. We also require $a_{1}^{1}+\gamma_{1}^{11}<0$. For example, if $\gamma_{1}^{11} \leq 0$, then there is either no autocatalytic action of species- 1 on itself, or a self-interference effect (intra-specific competition). If $a_{1}^{1}<0$, then $a_{1}^{1}+\gamma_{1}^{11}<0$ is satisfied in either case. Here, as discussed above, $\rho_{1}=-a_{1}^{1}$ can be interpreted as the linear death rate of species- 1 , which may be enhanced by self-interference if $\gamma_{1}^{11}<0$. The quadratic term $2 \gamma_{1}^{01} x_{0} x_{1}$ can be interpreted as the rate of growth of species-1 due to utilization of available substrate (e.g. through endogenous recruitment).

Because $g_{1}\left(x_{1}\right)$ is a quadratic in $x_{1}$, being inward pointing is sufficient to guarantee the existence and global stability of a unique interior equilibrium $\bar{x}_{1} \in(0,1)$. However, this is clearly not the case in higher dimensions (more than 1 species).

Example 2 (2-species systems). In the notation of (10), a 2-species system has the form

$$
\begin{aligned}
& g_{1}\left(x_{1}, x_{2}\right)=c_{1}+A_{1}^{1} x_{1}+A_{1}^{2} x_{2}+\Gamma_{1}^{11} x_{1}^{2}+2 \Gamma_{1}^{12} x_{1} x_{2}+\Gamma_{1}^{22} x_{2}^{2}, \\
& g_{2}\left(x_{1}, x_{2}\right)=c_{2}+A_{2}^{1} x_{1}+A_{2}^{2} x_{2}+\Gamma_{2}^{11} x_{1}^{2}+2 \Gamma_{2}^{12} x_{1} x_{2}+\Gamma_{2}^{22} x_{2}^{2} .
\end{aligned}
$$

There are three inward-pointing conditions: $\dot{x}_{i}>0$ when $x_{i}=0$ for $i=0,1,2$. These give three quadratic conditions

$$
\begin{aligned}
q_{1}(x)= & c_{1}+A_{1}^{2} x+\Gamma_{1}^{22} x^{2}>0, \\
q_{2}(x)= & c_{2}+A_{2}^{1} x+\Gamma_{2}^{11} x^{2}>0, \\
q_{0}(x)= & \left(c_{1}+c_{2}+A_{1}^{2}+A_{2}^{2}+\Gamma_{1}^{22}+\Gamma_{2}^{22}\right) \\
& +\left\{A_{1}^{1}+A_{2}^{1}-A_{1}^{2}-A_{2}^{2}+2\left(\Gamma_{1}^{12}+\Gamma_{2}^{12}\right)-2\left(\Gamma_{1}^{22}+\Gamma_{2}^{22}\right)\right\} x \\
& +\left\{\Gamma_{1}^{11}+\Gamma_{2}^{11}-2\left(\Gamma_{1}^{12}+\Gamma_{2}^{12}\right)+\Gamma_{1}^{22}+\Gamma_{2}^{22}\right\} x^{2}<0,
\end{aligned}
$$

for $0 \leq x \leq 1$. In particular, (12a) is satisfied if $c_{1}>0, c_{1}+A_{1}^{2}+\Gamma_{1}^{22}>0$ and $\Gamma_{1}^{22} \leq 0$. Similarly, (12b) is satisfied if $c_{2}>0, c_{2}+A_{2}^{1}+\Gamma_{2}^{11}>0$ and $\Gamma_{2}^{11} \leq 0$. Also, (12c) is satisfied if $c_{1}+c_{2}+A_{1}^{2}+A_{2}^{2}+\Gamma_{1}^{22}+\Gamma_{2}^{22}<0, c_{1}+c_{2}+A_{1}^{1}+A_{1}^{2}+\Gamma_{1}^{11}+\Gamma_{2}^{11}<0$ and $\Gamma_{1}^{11}+\Gamma_{2}^{11}-2\left(\Gamma_{1}^{12}+\Gamma_{2}^{12}\right)+\Gamma_{1}^{22}+\Gamma_{2}^{22} \geq 0$. 


\section{Equilibria}

\subsection{The index, uniqueness and global stability}

Consider a $C^{1}$ vector field $g$, defined on an open set in $\mathbf{R}^{n}$ containing $\Delta$, and satisfying the inward-pointing condition on $\partial \Delta$. The inward-pointing condition implies that there exists a sufficiently small $\delta>0$ such that $f(x)=x+\delta g(x) \in \Delta$ whenever $x \in \Delta$ (because $\Delta$ is compact). Notice that $f: \Delta \rightarrow \Delta$ is a continuous map, and hence has at least one fixed point in $\Delta$ by Brouwer's Fixed Point Theorem (Milnor, 1965). Fixed points of $f$ are the same as zeros of $g$ and hence they are equilibria of the dynamical system $\dot{x}=g(x)$. Further, since $g(x)$ is nowhere zero on $\partial \Delta$, every equilibrium lies in the interior of $\Delta$.

Recall that $x \in \Delta$ is a regular point if $\operatorname{det} \operatorname{Dg}(x) \neq 0$. By Sard's Theorem, almost all points are regular (Milnor, 1965). We make the following genericity assumption.

Assumption 1. Every equilibrium of $g(x)$ in $\Delta$ is regular.

This implies that $g(x)$ is injective in a neighbourhood of an equilibrium by the Inverse Function Theorem, and hence that every equilibrium is isolated. Assumption 1 almost always holds for naturally arising systems, which are generic. If it does not hold, then an $\varepsilon$-perturbation of the coefficients of $g$ will suffice to obtain the assumption.

Given a (regular) equilibrium $\bar{x} \in \operatorname{int} \Delta$, the index of $\bar{x}$ is defined by $\operatorname{ind}_{g}(\bar{x})=$ sign det $D g(\bar{x})$ (Milnor, 1965; Hofbauer and Sigmund, 1998). Note that the vector field $-g(x)$ points outwards on $\partial \Delta$. Of course, this has the same equilibria as $g(x)$, and it is clear that ind ${ }_{-g}(\bar{x})=(-1)^{n}$ ind $_{g}(\bar{x})$. The Poincaré-Hopf Theorem applies to outwardpointing vector fields, and states that, for such vector fields,

$$
\sum_{\text {equilibria } \bar{x} \in \Delta} \operatorname{ind}_{-g}(\bar{x})=\chi(\Delta),
$$

where $\chi(\Delta)$ is the Euler-characteristic of $\Delta$ (Milnor, 1965). Since $\Delta$ is a simplex, $\chi(\Delta)=1$, and hence $I_{g}=\sum_{\bar{x}} \operatorname{ind}_{g}(\bar{x})=(-1)^{n}$. It now follows that there can be only an odd number of (regular) equilibria in $\Delta$.

We wish to find conditions under which there is exactly one equilibrium. This is clearly true if sign det $D g(\bar{x})$ is constant on $\Delta$ (either +1 or -1 for all $x \in \Delta$ ). For then all equilibria have the same index, and $I_{g}= \pm m=(-1)^{n}$, where $m$ is the number of equilibria in $\Delta$, and +1 or -1 is their common index. It follows that $m=1$ and $\operatorname{ind}_{g}(\bar{x})=(-1)^{n}$ for the unique equilibrium $\bar{x}$. We therefore look for conditions under which sign $\operatorname{det} D g(x)$ is a non-zero constant on $\Delta$. This Index Theorem implies existence and uniqueness of an interior equilibrium. However, more than this, we seek conditions under which all the eigenvalues of $D g(x)$ have negative real parts. Clearly this implies that $D g(x)$ is nonsingular and sign det $D g(x)=(-1)^{n}$ for each $x \in \Delta$. However, although this implies that there is a unique interior equilibrium, it is not enough to imply that this equilibrium is globally stable. For this, we need the well-known sufficient condition for stability due to Lyapunov.

Theorem 1. Suppose that $g(x)$ is inward pointing on $\partial \Delta$ and that Assumption 1 holds. Suppose also that there is a real, symmetric, positive definite $n \times n$ matrix $Q$ such that 
$Q D g(x)$ is negative definite on $\mathbf{R}^{n}$ for all $x \in \Delta$. Then there is a unique equilibrium $\bar{x} \in$ int $\Delta$ of the system $\dot{x}=g(x)$, and $\bar{x}$ is globally asymptotically stable.

Indeed, that $Q D g(x)$ is negative definite implies that $D g(x)$ has eigenvalues with negative real parts, and hence that $\bar{x}$ exists and is unique by the Index Theorem above. In addition, $L(x)=\frac{1}{2} g(x) \cdot Q g(x)$ is a global Lyapunov function for $\bar{x}$, which yields global stability.

Note that the criterion of Theorem 1 is uniform in the sense that $Q$ must be a constant matrix, independent of $x$. It is elementary that a generic real matrix $A$ has eigenvalues with negative real parts if and only if there is a real, symmetric, positive definite matrix $Q$ such that $Q A$ is negative definite. However, the uniformity requirement of Theorem 1 is much stronger than this.

Remark 1. In fact, the existence of a $Q$ satisfying the conditions of Theorem 1 is itself sufficient to imply both the uniqueness of equilibrium and global stability, without appeal to the Index Theorem. Here we have chosen to separate the issue of the existence of a unique equilibrium from that of its global stability. Hence our appeal to the Index Theorem.

\subsection{Semi-neutral systems}

Semi-neutral systems provide an example in which the uniformity assumption on $Q$ in Theorem 1 can be weakened. By a semi-neutral system we mean a system of the form (6) in which the $n$ species do not interact directly with each other but compete only for the underlying substrate. However, we allow the possibility of intra-specific competition. If there is no intra-specific competition, the system is strictly neutral. Thus, in a semineutral system $a_{i}^{j}=0$ for $j \neq i, 1 \leq j \leq n$, and $\gamma_{i}^{j k}=0$ for all $0 \leq j, k \leq n$, except possibly the substrate interaction coefficients $\gamma_{i}^{i 0}$ and the self-interaction coefficients $\gamma_{i}^{i i}$. Assume that $a_{i}^{i}=-\rho_{i}$, where $\rho_{i}>0$ is the death (or emigration) rate of species $i$, and also that $2 \gamma_{i}^{i 0}=\lambda_{i}$. As discussed in Section 2, if $\lambda_{i}>0$, this can be interpreted as the growth rate of established species $i$ due to utilization of available substrate, and if $\lambda_{i}<0$, it can be interpreted as a density effect, with established species $i$ inhibiting recruitment and/or growth of new representatives. We write $\kappa_{i}=-\gamma_{i}^{i i}$ and assume that $\kappa_{i} \geq 0$, so that this coefficient represents a self-inhibitory term. We interpret $a_{i}^{0}>0$ as the intrinsic exogenous recruitment rate of species $i$ onto available substrate. The system (6) therefore reduces to the form

$$
\dot{x}_{i}=a_{i}^{0} x_{0}-\rho_{i} x_{i}+\lambda_{i} x_{i} x_{0}-\kappa_{i} x_{i}^{2} \quad(1 \leq i \leq n) .
$$

This can be written as $\dot{x}_{i}=\left(a_{i}^{0}+\lambda_{i} x_{i}\right) x_{0}-\rho_{i} x_{i}-\kappa_{i} x_{i}^{2}$, and we assume that the net substrate utilization rate $a_{i}^{0}+\lambda_{i} x_{i}$ is always positive (even when $\lambda_{i}<0$ ). This is the case if $a_{i}^{0}>-\lambda_{i}$. Also, (13) can be written as $\dot{x}_{i}=a_{i}^{0} x_{0}-\left(\rho_{i}-\lambda_{i} x_{0}\right) x_{i}-\kappa_{i} x_{i}^{2}$. Assume that species $i$ cannot persist without exogenous recruitment $\left(a_{i}^{0}=0\right)$. This is the case if $\rho_{i}-\lambda_{i} x_{0}$ is always positive (even when $\lambda_{i}>0$ ). Thus, assume that $\rho_{i}>\lambda_{i}$. Clearly, 
$\dot{x}_{i}>0$ when $x_{i}=0$, provided there is exogenous recruitment $\left(a_{i}^{0}>0\right)$ and some available substrate $\left(x_{0}>0\right)$. Also,

$$
\dot{x}_{0}=-\sum_{i=1}^{n} \dot{x}_{i}=(\rho \cdot x)+\left(\kappa \cdot x^{2}\right)-\left(a_{0}+(\lambda \cdot x)\right) x_{0},
$$

where $a_{0}=\sum_{i=1}^{n} a_{i}^{0}$. It follows that $\dot{x}_{0}>0$ when $x_{0}=0$. The inward-pointing conditions are therefore satisfied.

Define a positive definite, diagonal matrix $Q(x)=\operatorname{diag}\left\{d_{1}\left(x_{1}\right), \ldots, d_{n}\left(x_{n}\right)\right\}$, by taking

$$
d_{i}\left(x_{i}\right)=\frac{1}{a_{i}^{0}+\lambda_{i} x_{i}} .
$$

It is shown in Appendix A that $Q(x) D g(x)$ is negative definite for all $x \in \Delta$, from which it follows that the eigenvalues of $D g(x)$ all have negative real parts. Hence, from the Index Theorem (Section 3.1), there is a unique equilibrium $\bar{x} \in$ int $\Delta$. Further, we show in Appendix A that $L(x)=\frac{1}{2} g(x) \cdot Q(x) g(x)$ is a global Lyapunov function for the system, and hence that $\bar{x}$ is globally asymptotically stable.

Observe from (13) that the unique equilibrium satisfies the equations

$$
\bar{x}_{i}=\frac{1}{2 \kappa_{i}}\left\{-\left(\rho_{i}-\lambda_{i} \bar{x}_{0}\right)+\sqrt{\left(\rho_{i}-\lambda_{i} \bar{x}_{0}\right)^{2}+4 \kappa_{i} a_{i}^{0} \bar{x}_{0}}\right\} \quad(1 \leq i \leq n) .
$$

Since $\rho_{i}>\lambda_{i}$, it follows that $\bar{x}_{i} \rightarrow 0$ as $a_{i}^{0} \rightarrow 0$. Thus, if there is no exogenous recruitment $\left(a_{i}^{0}=0\right)$, then species $i$ is eliminated by competitive exclusion. This shows that, under the given assumptions, the non-zero equilibrium representation of any of the species depends essentially on exogenous recruitment.

\section{Diagonal dominance}

In this section we recall the basic theory of diagonal dominance for an $n \times n$ matrix. We then extend this to define a notion of uniform diagonal dominance for a matrix-valued function. This is used to determine explicit conditions under which a general differentiable dynamical system $\dot{x}=g(x)$, defined on a simplex, admits a unique, globally stable equilibrium. These conditions are then applied to quadratic systems of the form (10).

\subsection{Diagonal dominance}

Let $M_{n}(\mathbf{R})$ be the space of real, $n \times n$ matrices, and $M_{n}^{+}(\mathbf{R}) \subset M_{n}(\mathbf{R})$ the positive cone of those matrices having positive diagonal elements. For $\Omega \in M_{n}(\mathbf{R})$, define the derived matrix $\hat{\Omega} \in M_{n}(\mathbf{R})$ by: $\hat{\omega}_{i i}=\left|\omega_{i i}\right|$ and $\hat{\omega}_{i j}=-\left|\omega_{i j}\right|$ for $i \neq j$. Thus, $\hat{\Omega}$ has non-negative diagonal entries, and non-positive off-diagonal entries. Clearly, $\hat{\Omega} \in M_{n}^{+}(\mathbf{R})$ if $\Omega \in M_{n}^{+}(\mathbf{R})$, and in this case $\hat{\omega}_{i i}=\omega_{i i}$. For vectors $u, v \in \mathbf{R}^{n}$ we write $u>v$ (resp. $u \geq v$ ) to mean $u_{i}>v_{i}$ (resp. $u_{i} \geq v_{i}$ ) for $1 \leq i \leq n$. A vector $u$ is said to be positive (resp. non-negative) if $u>0$ (resp. $u \geq 0$ ). 
Recall that $\Omega \in M_{n}(\mathbf{R})$ has a row-dominant diagonal if there exists a positive vector $d=\left(d_{1}, \ldots, d_{n}\right)^{T} \in \mathbf{R}^{n}$ such that $\hat{\Omega} d>0$. Explicitly, this means that there are positive constants $d_{1}, \ldots, d_{n}$ such that

$$
d_{i}\left|\omega_{i i}\right|>\sum_{j \neq i} d_{j}\left|\omega_{i j}\right|, \quad 1 \leq i \leq n
$$

A matrix $\Omega$ has a column-dominant diagonal if its transpose, $\Omega^{T}$, has a row-dominant diagonal, that is, if there exists $c>0$ such that $\Omega^{T} c>0$. Equivalently:

$$
c_{i}\left|\omega_{i i}\right|>\sum_{j \neq i} c_{j}\left|\omega_{j i}\right|, \quad 1 \leq i \leq n .
$$

A key result concerning matrices with a dominant diagonal (either row or column) is:

Proposition 1. If $\Omega \in M_{n}(\mathbf{R})$ has a dominant diagonal then it is non-singular. If, in addition, $\Omega \in M_{n}^{+}(\mathbf{R})$, then the eigenvalues of $\Omega$ all have positive real parts.

See Takayama (1985, Chap. 4, Theorems 4.C.1 and 4.C.2).

The row-dominant diagonal condition $\hat{\Omega} d>0$ is equivalent to the so-called HawkinsSimon $(H-S)$ conditions for $\hat{\Omega}$ :

$$
\hat{\Omega}_{(k)}=\operatorname{det}\left(\begin{array}{cccc}
\hat{\omega}_{11} & \hat{\omega}_{12} & \cdots & \hat{\omega}_{1 k} \\
\hat{\omega}_{21} & \hat{\omega}_{22} & \cdots & \hat{\omega}_{2 k} \\
\vdots & \vdots & & \vdots \\
\hat{\omega}_{k 1} & \hat{\omega}_{k 2} & \cdots & \hat{\omega}_{k k}
\end{array}\right)>0 \quad \text { for } 1 \leq k \leq n .
$$

That is, $\hat{\Omega}$ is an $M$-matrix (a matrix with non-positive off-diagonal entries all of whose successive principal minors are positive). See Takayama (1985, Chap. 4, Theorem 4.C.5). In fact, the $\mathrm{H}-\mathrm{S}$ conditions imply that all the principal minors of $\hat{\Omega}$ are positive (i.e. the determinants of the submatrices obtained by choosing elements $\hat{\omega}_{i j}$ for $i, j$ in any non-empty subset $S \subseteq\{1,2, \ldots, n\})$. See Takayama (1985, Chap. 4, Corollary to Theorem 4.C.5). In particular, $\left|\omega_{i i}\right|=\hat{\omega}_{i i}>0$ for $1 \leq i \leq n$.

Remark 2. Clearly $\hat{\Omega}$ is an $M$-matrix if and only if $\hat{\Omega}^{T}$ is an $M$-matrix. It therefore follows that row-diagonal dominance and column-diagonal dominance are equivalent. If $\Omega \in M_{n}^{+}(\mathbf{R})$ (i.e. has positive diagonal entries), the row- or column-diagonal dominance conditions suffice to ensure that there exists a positive diagonal matrix $Q$ such that $Q \Omega$ is positive definite—see Araki and Kondo (1972), Ikeda and Siljak (1980), and Theorem 2 below.

\subsection{Uniform diagonal dominance}

Let $\Omega: \Delta \rightarrow M_{n}(\mathbf{R})$ be a continuous, matrix-valued function. The derived function $\hat{\Omega}$ : $\Delta \rightarrow M_{n}(\mathbf{R})$ is then also continuous, and takes values in $M_{n}^{+}(\mathbf{R})$ if $\Omega$ does. 
We say that $\Omega$ has a uniformly row-dominant diagonal if there exists a vector $d>0$ such that $\hat{\Omega}(x) d>0$ for all $x \in \Delta$. Explicitly, there are positive constants $d_{1}, \ldots, d_{n}$ (independent of $x$ ) such that

$$
f_{i}(x)=d_{i}\left|\omega_{i i}(x)\right|-\sum_{j \neq i} d_{j}\left|\omega_{i j}(x)\right|>0
$$

for all $x \in \Delta$ and $1 \leq i \leq n$. If $\Omega^{T}: \Delta \rightarrow M_{n}(\mathbf{R})$ is the transpose function defined by $\Omega^{T}(x)=\Omega(x)^{T}$, then $\Omega$ has a uniformly column-dominant diagonal if $\Omega^{T}$ has a uniformly row-dominant diagonal, that is, if there exists a constant vector $c>0$ such that $\Omega^{T}(x) c>0$. Note that if $\Omega$ has a uniformly (row or column) dominant diagonal, then $\hat{\Omega}$ and $\hat{\Omega}^{T}$ take values in $M_{n}^{+}(\mathbf{R})$, whether or not $\Omega$ does.

Remark 3. Note that $\Omega(x)$ having a row-dominant diagonal implies that it also has a column-dominant diagonal (Remark 2). However, there is no reason to suppose that $\Omega(x)$ having a uniformly row-dominant diagonal implies that it has a uniformly columndominant diagonal (or vice versa). In particular, if $\Omega$ takes values in $M_{n}^{+}(\mathbf{R})$, we cannot conclude that there is a constant positive diagonal matrix $Q$ such that $Q \Omega(x)$ is positive definite for all $x$.

To apply these notions to a dynamical system $\dot{x}=g(x)$ on a neighbourhood of $\Delta$, consider the Jacobian matrix. Thus, if $g(x)$ is quadratic we can write $-D g(x)$ in the form

$$
-D g(x)=\Omega(x)=\sum_{r=0}^{n} \Omega^{(r)} x_{r}, \quad \Omega^{(r)}=\left(\omega_{i j}^{(r)}\right), \quad 0 \leq r \leq n .
$$

In spite of the fact that row- and column-diagonal dominance are not in general equivalent, if it happens to be true that $\Omega$ has both a uniformly row- and a uniformly columndominant diagonal, then it is the case that a suitable $Q$ can be found for the application of Theorem 1. This is shown in the following theorem, proved in Appendix A.

Theorem 2. Let $g(x)$ be a $C^{1}$ vector field defined on a neighbourhood of $\Delta$ in $\mathbf{R}^{n}$ that is inward-pointing on $\partial \Delta$ and which satisfies Assumption 1. Suppose that $\Omega=-D g: \Delta \rightarrow$ $M_{n}^{+}(\mathbf{R})$ has both a uniformly row-and a uniformly column-dominant diagonal. Then there is a positive diagonal matrix $Q$ such that $Q D g(x)$ is negative definite on $\Delta$. Hence there is a unique equilibrium $\bar{x} \in$ int $\Delta$ of the system $\dot{x}=g(x)$, and $\bar{x}$ is globally asymptotically stable.

A stronger theorem than Theorem 2 can be proved under the assumption that $\Omega$ has either a uniform row- or column-dominant diagonal, but not necessarily both. This does not rely on finding a symmetric, positive definite matrix $Q$ as in Theorem 2. Instead, different Lyapunov functions are used than that used in Theorem 1 . The proof of the following theorem is given in Appendix A.

Theorem 3. Let $g(x)$ be a $C^{1}$ vector field defined on a neighbourhood of $\Delta$ in $\mathbf{R}^{n}$ that is inward-pointing on $\partial \Delta$ and which satisfies Assumption 1. Suppose that $\Omega=-D g: \Delta \rightarrow$ $M_{n}^{+}(\mathbf{R})$ has either a uniformly row-dominant diagonal or a uniformly column-dominant 
diagonal. Then there is a unique equilibrium $\bar{x} \in$ int $\Delta$ of the system $\dot{x}=g(x)$, and $\bar{x}$ is globally asymptotically stable.

\subsection{General conditions for uniform diagonal dominance}

We now consider conditions under which $\Omega: \Delta \rightarrow M_{n}(\mathbf{R})$ has a uniformly dominant diagonal. We develop these conditions for row dominance, but analogous conditions for column dominance can be obtained by replacing $\Omega$ by $\Omega^{T}$.

Let $e_{0}=0 \in \mathbf{R}^{n}$ be the vector obtained by taking $x_{1}=\cdots=x_{n}=0$ (equivalently, $x_{0}=1$ ), and $e_{r}$ the vector obtained by taking $x_{r}=1$ (and $x_{s}=0$ for $s \neq r$ ) for $1 \leq r \leq n$.

Proposition 2. Let $\Omega: \Delta \rightarrow M_{n}(\mathbf{R})$ be continuous. Suppose that $\left|\omega_{i i}(x)\right|$ is a concave function of $x \in \Delta$, and $\left|\omega_{i j}(x)\right|$ is a convex function of $x \in \Delta$ for $i \neq j$. Then $f_{i}(x)$ defined in (18) takes its minimum value $\underline{f}_{i}=\inf _{x \in \Delta} f_{i}(x)$ at (at least) one of the extreme points $x=e_{0}, e_{1}, \ldots, e_{n}$, that is,

$$
\underline{f}_{i}=\min \left\{f_{i}\left(e_{0}\right), f_{i}\left(e_{1}\right), \ldots, f_{i}\left(e_{n}\right)\right\} .
$$

Proof: Write $f_{i}(x)=h_{i}(x)-k_{i}(x)$, where $h_{i}(x)=d_{i}\left|\omega_{i i}(x)\right|$, and $k_{i}(x)=$ $\sum_{j \neq i} d_{j}\left|\omega_{i j}(x)\right|$. By assumption, $h_{i}(x)$ is concave and $k_{i}(x)$ is convex, and hence $f_{i}(x)$ is concave. We may write $x \in \Delta$ as a convex combination of the extreme points of $\Delta$; i.e. $x=x_{0} e_{0}+x_{1} e_{1}+\cdots+x_{n} e_{n}$, with $x_{0}+x_{1}+\cdots+x_{n}=1$. Then $f_{i}(x)$ being concave implies that $f_{i}(x) \geq x_{0} f_{i}\left(e_{0}\right)+x_{1} f_{i}\left(e_{1}\right)+\cdots+x_{n} f_{i}\left(e_{n}\right)$, and (20) follows immediately.

Under the conditions of Proposition 2, it follows that $\Omega(x)$ has a uniformly rowdominant diagonal provided there is a $d>0$ for which $\underline{f}_{i}>0$ for all $1 \leq i \leq n$. From (18) and (20) $\underline{f}_{i}$ has the form

$$
\underline{f}_{i}=d_{i} f_{i i}-\sum_{j \neq i} d_{j} f_{i j},
$$

with $f_{i i}>0$ and $f_{i j} \geq 0$. Thus, $f_{i}>0$ for all $i$ is equivalent to the matrix $F=\left(f_{i j}\right)$ having a row-dominant diagonal. This in turn is equivalent to the H-S conditions (17) for the matrix $\hat{F}=\left(\hat{f}_{i j}\right)$. Thus, if we can identify the $f_{i j}$ in terms of components of $\Omega$, to show uniform row-diagonal dominance, we are reduced to verifying a set of $n$ determinant conditions for the derived matrix $\hat{F}$.

\subsection{Explicit conditions for uniform diagonal dominance}

To proceed further involves identifying, for each $i$, which of the extreme values $f_{i}\left(e_{r}\right)$ determine the minimum $\underline{f}_{i}$ (see (20)). In principle, this may be different for different $d$. However, we shall seek more specialized conditions under which the minimizing extreme value is the same for all $d>0$. Such conditions are provided by the following proposition.

Proposition 3. Let $\Omega: \Delta \rightarrow M_{n}(\mathbf{R})$ satisfy the assumptions of Proposition 2, and write $\Omega\left(e_{r}\right)=\Omega^{(r)}=\left(\omega_{i j}^{(r)}\right)$ for $0 \leq r \leq n$. Then sufficient conditions that $\underline{f}_{i}=f_{i}\left(e_{r}\right)$ for all 
$d>0$ are:

$$
\begin{aligned}
& \left|\omega_{i i}^{(s)}\right| \geq\left|\omega_{i i}^{(r)}\right| \quad \text { for } 0 \leq s \leq n, \\
& \left|\omega_{i j}^{(r)}\right| \geq\left|\omega_{i j}^{(s)}\right| \quad \text { for } 1 \leq j \leq n, j \neq i \text { and } 0 \leq s \leq n .
\end{aligned}
$$

Proof: $\underline{f}_{i}=f_{i}\left(e_{r}\right)$ for all $d>0$ only if $\ell_{i}^{(r, s)}(d)=f_{i}\left(e_{s}\right)-f_{i}\left(e_{r}\right) \geq 0$ for $0 \leq s \leq n$ and all $d>0$. But, from (18), $f_{i}\left(e_{r}\right)=d_{i}\left|\omega_{i i}^{(r)}\right|-\sum_{j \neq i} d_{j}\left|\omega_{i j}^{(r)}\right|$. Hence,

$$
\ell_{i}^{(r, s)}(d)=d_{i}\left\{\left|\omega_{i i}^{(s)}\right|-\left|\omega_{i i}^{(r)}\right|\right\}+\sum_{j \neq i} d_{j}\left\{\left|\omega_{i j}^{(r)}\right|-\left|\omega_{i j}^{(s)}\right|\right\}
$$

It follows that $\ell_{i}^{(r, s)}(d) \geq 0$ for all $d>0$ if conditions (22) hold.

If the conditions of the form (22) hold for each $i$, then we can identify an $r(i) \in$ $\{0,1, \ldots, n\}$ such that $f_{i}=f_{i}\left(e_{r(i)}\right)$ for all $d>0$ and $1 \leq i \leq n$. This defines a function $r:\{1,2, \ldots, n\} \rightarrow\{0, \overline{1}, 2, \ldots, n\}$. Given such a function, the coefficients $f_{i j}$ in (21) are precisely identified, and so the $n \mathrm{H}-\mathrm{S}$ determinant conditions (17) for the associated matrix $\hat{F}=\left(\hat{f}_{i j}\right)$ can be evaluated. If these conditions are satisfied, together with the assumptions of Proposition 2, then we can conclude that $A$ has a uniformly row-dominant diagonal. However, as noted in Remark 3, uniform row-diagonal dominance does not in general imply uniform column-diagonal dominance. In particular, Theorem 2 cannot be applied.

Nevertheless, under some circumstances, uniform row and column diagonal dominance are equivalent, and hence Theorem 2 can be applied. The simplest case occurs when $r$ is a constant function; i.e. $r(i)=m$ for some $m \in\{0,1, \ldots, n\}$ and all $1 \leq i \leq n$. In this case, $\hat{f}_{i i}=\left|\omega_{i i}^{(m)}\right|$ and $\hat{f}_{i j}=-\left|\omega_{i j}^{(m)}\right|$ for $j \neq i$, and conditions (22) are: $\left|\omega_{i i}^{(s)}\right| \geq\left|\omega_{i i}^{(m)}\right|$ for $1 \leq i \leq n$ and $0 \leq s \leq n$, and $\left|\omega_{i j}^{(m)}\right| \geq\left|\omega_{i j}^{(s)}\right|$ for $1 \leq i, j \leq n, j \neq i$ and $0 \leq s \leq n$. If the H-S conditions (17) also hold for $\hat{F}=\left(\hat{f}_{i j}\right)$, then we conclude that $\Omega$ has a uniformly row-dominant diagonal. However, conditions (22) also imply that $\left|\omega_{j i}^{(m)}\right| \geq\left|\omega_{j i}^{(s)}\right|$ for $1 \leq i, j \leq n, j \neq i$ and $0 \leq s \leq n$, and the H-S conditions for $\hat{F}$ are equivalent to the H-S conditions for $\hat{F}^{T}$. Thus, Propositions 2 and 3 imply that $\Omega$ has both a uniformly row and a uniformly column-dominant diagonal. Theorem 2 can then be applied to obtain:

Theorem 4. Let $g(x)$ be a $C^{1}$ vector field defined on a neighbourhood of $\Delta$ in $\mathbf{R}^{n}$ that is inward-pointing on $\partial \Delta$ and which satisfies Assumption 1. Let $\Omega=-D g(x): \Delta \rightarrow$ $M_{n}^{+}(\mathbf{R})$, and suppose that $\omega_{i i}(x)$ is a concave function of $x \in \Delta$ for $1 \leq i \leq n$, and $\left|\omega_{i j}(x)\right|$ is a convex function of $x \in \Delta$ for $1 \leq i, j \leq n, j \neq i$. Assume there is an $m \in\{0,1, \ldots, n\}$ such that $\omega_{i i}^{(s)} \geq \omega_{i i}^{(m)}$ for $1 \leq i \leq n, 0 \leq s \leq n$, and $\left|\omega_{i j}^{(m)}\right| \geq\left|\omega_{i j}^{(s)}\right|$ for $1 \leq i, j \leq n, j \neq i$ and $0 \leq s \leq n$. Suppose also that the $H$-S conditions (17) hold for the matrix $\hat{F}=\left(\hat{f}_{i j}\right)$ with entries $\hat{f}_{i i}=\omega_{i i}^{(m)}$ and $\hat{f}_{i j}=-\left|\omega_{i j}^{(m)}\right|$ for $j \neq i$. Then there is a positive diagonal matrix $Q$ such that is $Q D g(x)$ is negative definite on $\Delta$. Hence, there is a unique equilibrium $\bar{x} \in$ int $\Delta$ of the system $\dot{x}=g(x)$, and $\bar{x}$ is globally asymptotically stable.

In the more general case in which $r:\{1,2, \ldots, n\} \rightarrow\{0,1,2, \ldots, n\}$ is not a constant function, Theorem 3 can be applied to obtain Theorem 5 below. We state the result 
for row-diagonal dominance, but an analogous statement can be obtained for columndiagonal dominance by transposition.

Theorem 5. Let $g(x)$ be a $C^{1}$ vector field defined on a neighbourhood of $\Delta$ in $\mathbf{R}^{n}$ that is inward-pointing on $\partial \Delta$ and which satisfies Assumption 1 . Let $\Omega=-D g(x): \Delta \rightarrow$ $M_{n}^{+}(\mathbf{R})$, and suppose that $\omega_{i i}(x)$ is a concave function of $x \in \Delta$ for $1 \leq i \leq n$, and $\left|\omega_{i j}(x)\right|$ is a convex function of $x \in \Delta$ for $1 \leq i, j \leq n, j \neq i$. Assume there is a function $r:\{1,2, \ldots, n\} \rightarrow\{0,1,2, \ldots, n\}$ such that $\omega_{i i}^{(s)} \geq \omega_{i i}^{(r(i))}$ for $1 \leq i \leq n, 0 \leq s \leq n$, and $\left|\omega_{i j}^{(r(i))}\right| \geq\left|\omega_{i j}^{(s)}\right|$ for $1 \leq i, j \leq n, j \neq i$ and $0 \leq s \leq n$. Suppose also that the H-S conditions (17) hold for the matrix $\hat{F}=\left(\hat{f}_{i j}\right)$ with entries $\hat{f}_{i i}=\omega_{i i}^{(r(i))}$ and $\hat{f}_{i j}=-\left|\omega_{i j}^{(r(i))}\right|$ for $j \neq i$. Then there is a unique equilibrium $\bar{x} \in$ int $\Delta$ of the system $\dot{x}=g(x)$, and $\bar{x}$ is globally asymptotically stable.

\section{Applications to quadratic systems}

In this section, we apply the preceding discussion to quadratic systems of the form (10). To do this we require that $\Omega=-D g$ take values in $M_{n}^{+}(\mathbf{R})$. From (10),

$$
D_{i j} g(x)=\frac{\partial g_{i}(x)}{\partial x_{j}}=A_{i}^{j}+2 \sum_{r=1}^{n} \Gamma_{i}^{j r} x_{r}=A_{i}^{j} x_{0}+\sum_{r=1}^{n}\left(A_{i}^{j}+2 \Gamma_{i}^{j r}\right) x_{r},
$$

and hence, comparing with (19),

$$
\omega_{i j}^{(0)}=-A_{i}^{j}, \quad \omega_{i j}^{(r)}=-\left(A_{i}^{j}+2 \Gamma_{i}^{j r}\right) \quad \text { for } 1 \leq r \leq n .
$$

To apply the theory of previous sections, we require the diagonal entries $\omega_{i i}^{(r)}$ to be positive for $1 \leq i \leq n, 0 \leq r \leq n$. We therefore make the following assumption.

Assumption 2. The coefficients $A_{i}^{i}$ and $A_{i}^{i}+2 \Gamma_{i}^{i r}$ are negative for $1 \leq i, r \leq n$.

It now follows from (23) that $\omega_{i i}(x)=\sum_{r=0}^{n} \omega_{i i}^{(r)} x_{r}>0$, and hence that $\Omega: \Delta \rightarrow$ $M_{n}^{+}(\mathbf{R})$.

Suppose the quadratic system $g(x)$ defined by (10) is inward pointing on $\partial \Delta$ and that Assumptions 1 and 2 hold for $g(x)$. Suppose also that there is a (constant) real, symmetric, positive definite matrix $Q$ such that $Q D g(x)$ is negative definite on $\mathbf{R}^{n}$. From (19), this implies that the quadratic form on $\mathbf{R}^{n}$ given by $q(u, x)=u \cdot Q \Omega(x) u$ is positive definite for each $x \in \Delta$. This is the case if and only if $q^{(r)}(u)=u \cdot Q \Omega^{(r)} u$ is positive definite for $0 \leq r \leq n$. Applying (19) and (23) together with Theorem 1 to this situation therefore yields:

Theorem 6. Suppose that the vector field $g(x)$ defined by (10) is inward-pointing on $\partial \Delta$ and that Assumptions 1 and 2 hold. Let $A=\left(A_{i}^{j}\right)$ and $G_{r}=\left(A_{i}^{j}+2 \Gamma_{i}^{j r}\right)$ for $1 \leq r \leq n$, and suppose that there is a real, symmetric, positive definite $n \times n$ matrix $Q$ such that $Q A$ and $Q G_{r}$ are negative definite on $\mathbf{R}^{n}$ for $1 \leq r \leq n$. Then there is a unique equilibrium $\bar{x} \in$ int $\Delta$ of the system $\dot{x}=g(x)$, and $\bar{x}$ is globally asymptotically stable. 
Example 3 (2-species systems). For the 2-species system (11), assume that $g\left(x_{1}, x_{2}\right)$ is inward pointing on $\partial \Delta$. The $2 \times 2$ matrices $A, G_{1}, G_{2}$ are

$$
A=\left(\begin{array}{ll}
A_{1}^{1} & A_{1}^{2} \\
A_{2}^{1} & A_{2}^{2}
\end{array}\right), \quad G_{r}=\left(\begin{array}{cc}
A_{1}^{1}+2 \Gamma_{1}^{1 r} & A_{1}^{2}+2 \Gamma_{1}^{2 r} \\
A_{2}^{1}+2 \Gamma_{2}^{1 r} & A_{2}^{2}+2 \Gamma_{2}^{2 r}
\end{array}\right) \quad(r=1,2) .
$$

Suppose there is a positive diagonal matrix $Q=\operatorname{diag}\left\{d_{1}, d_{2}\right\}$ such that $Q A, Q G_{1}, Q G_{2}$ are negative definite. This is the case if and only if the symmetric parts of these matrices are negative definite: i.e., if and only if

$$
\begin{aligned}
& \sigma(Q A)=\left(\begin{array}{cc}
d_{1} A_{1}^{1} & \frac{1}{2}\left(d_{1} A_{1}^{2}+d_{2} A_{2}^{1}\right) \\
\frac{1}{2}\left(d_{1} A_{1}^{2}+d_{2} A_{2}^{1}\right) & d_{2} A_{2}^{2}
\end{array}\right), \\
& \sigma\left(Q G_{r}\right)=\left(\begin{array}{cc}
d_{1}\left(A_{1}^{1}+2 \Gamma_{1}^{1 r}\right) & \frac{1}{2}\left\{d_{1}\left(A_{1}^{2}+2 \Gamma_{1}^{2 r}\right)+d_{2}\left(A_{2}^{1}+2 \Gamma_{2}^{1 r}\right)\right\} \\
\frac{1}{2}\left\{d_{1}\left(A_{1}^{2}+2 \Gamma_{1}^{2 r}\right)+d_{2}\left(A_{2}^{1}+2 \Gamma_{2}^{1 r}\right)\right\} & d_{2}\left(A_{2}^{2}+2 \Gamma_{2}^{2 r}\right)
\end{array}\right)
\end{aligned}
$$

are negative definite. Each of these symmetric matrices has negative eigenvalues (and hence are negative definite) if and only if they have negative trace and positive determinant. A symmetric $2 \times 2$ matrix can have both these properties only if its diagonal entries are negative. We therefore obtain the following.

Proposition 4. For a 2-species ecosystem (11) satisfying the inward-pointing conditions (Example 2) and Assumption 1, suppose there are positive constants $d_{1}$ and $d_{2}$ such that:

$$
\begin{aligned}
& A_{i}^{i}, A_{i}^{i}+2 \Gamma_{i}^{i r}<0 \quad \text { for } 1 \leq i, r \leq 2, \\
& A_{1}^{1} A_{2}^{2}>\frac{1}{4 d_{1} d_{2}}\left(d_{1} A_{1}^{2}+d_{2} A_{2}^{1}\right)^{2}, \\
& \left(A_{1}^{1}+2 \Gamma_{1}^{1 r}\right)\left(A_{2}^{2}+2 \Gamma_{2}^{2 r}\right)>\frac{1}{4 d_{1} d_{2}}\left\{d_{1}\left(A_{1}^{2}+2 \Gamma_{1}^{2 r}\right)+d_{2}\left(A_{2}^{1}+2 \Gamma_{2}^{1 r}\right)\right\}^{2}, \\
& \quad \text { for } r=1,2 .
\end{aligned}
$$

Then the conclusion of Theorem 6 holds.

To apply Proposition 4, observe that it suffices to assume that $d_{1}+d_{2}=1$. If the lefthand sides of (24b), (24c) are $\ell^{(r)}$ and the right-hand sides are $h^{(r)}\left(d_{1}, d_{2}\right), 0 \leq r \leq 2$, we can define three sets:

$$
D^{(r)}=\left\{d=d_{1} \in(0,1): d_{1}+d_{2}=1 \text { and } \ell^{(r)}>h^{(r)}\left(d_{1}, d_{2}\right)\right\} \quad(0 \leq r \leq 2) .
$$

Then (24b), (24c) are satisfied simultaneously only if $\hat{D}=\bigcap_{r=0}^{2} D^{(r)}$ is non-empty. This situation is illustrated in Fig. 1.

Note that the left-hand sides $\ell^{(r)}, 0 \leq r \leq 2$, involve only the diagonal entries of the matrices $A, G_{1}, G_{2}$, and the right-hand sides $h^{(r)}\left(d_{1}, d_{2}\right)$ involve only the off-diagonal entries. Further, it is easy to show that $D^{(r)} \rightarrow(0,1)$ as $\ell^{(r)} \rightarrow \infty$. It follows that (24b), (24c) will always hold provided $\min _{r} \ell^{(r)}$ is sufficiently large. From (24a), for a fixed set of other parameters, this is the case if $A_{i}^{i}$ are sufficiently large and negative for $i=1,2$. Thus, by 


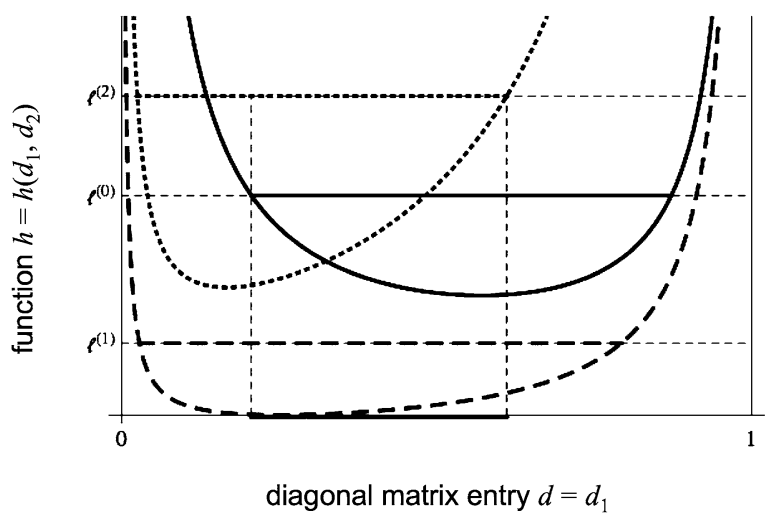

Fig. 1 The curves $h^{(r)}\left(d_{1}, d_{2}\right)$ for $d_{1}=d, d_{2}=1-d$ with $0<d<1$ and $r=0$ (solid black curve; right-hand side of (24b)), $r=1$ (long-dashed curve; right-hand side of (24c)) and $r=2$ (dotted curve; right-hand side of $(24 \mathrm{c})$ ). The values $\ell^{(r)}$ on the vertical axis are the left-hand sides of inequalities (24b), (24c). The heavy horizontal line associated with each of these values shows the (open) range of values of $d$ for which the corresponding inequality holds; i.e. the sets $D^{(r)}, 0 \leq r \leq 2$. The intersection of these regions is $\hat{D}$, indicated by the heavy black line on the $d$-axis. This is the (non-empty) range of values of $d$, for which the three inequalities (24b), (24c) hold simultaneously

boosting these negative, self-limiting terms sufficiently (while leaving other terms fixed), the 2-species system can always be made globally asymptotically stable.

To apply Theorem 5 to quadratic systems, we consider explicit conditions under which $\Omega$ has a uniformly row-dominant dynamic for (10). Clearly, $\omega_{i i}(x)=\sum_{r=0}^{n} \omega_{i i}^{(r)} x_{r}$ is positive (by Assumption 2) and linear, and hence is a concave function of $x$. Similarly, $\omega_{i j}(x)=\sum_{r=0}^{n} \omega_{i j}^{(r)} x_{r}$ is linear, and hence $\left|\omega_{i j}(x)\right|$ is a convex function of $x$ for $j \neq i$. This shows that $\Omega$ satisfies the hypotheses of Proposition 2. We can therefore apply Proposition 3 to obtain explicit conditions (22) for quadratic systems. We distinguish two cases: $\underline{f}_{i}=f_{i}\left(e_{0}\right)$ and $\underline{f}_{i}=f_{i}\left(e_{r}\right)$ for $r \geq 1$. Using Assumption 2 and (22) we obtain the following.

Proposition 5. Under Assumption 2:

(i) Sufficient conditions that $\underline{f}_{i}=f_{i}\left(e_{0}\right)$ for any $d>0$ are:

$$
\begin{aligned}
& A_{i}^{i}<0, \\
& \Gamma_{i}^{i s} \leq 0 \quad \text { for } 1 \leq s \leq n, \\
& \text { If } \Gamma_{i}^{j s} \neq 0, \text { then } \operatorname{sign} A_{i}^{j}=-\operatorname{sign} \Gamma_{i}^{j s} \quad \text { for } j \neq i, 1 \leq s \leq n, \\
& \left|A_{i}^{j}\right| \geq\left|\Gamma_{i}^{j s}\right| \quad \text { for } j \neq i, 1 \leq s \leq n,
\end{aligned}
$$

(ii) Necessary and sufficient conditions that $\underline{f}_{i}=f_{i}\left(e_{r}\right)$ with $1 \leq r \leq n$, for any $d>0$ are:

$$
\begin{aligned}
& \Gamma_{i}^{i r} \geq 0, \\
& A_{i}^{i}+2 \Gamma_{i}^{i r}<0,
\end{aligned}
$$


$\Gamma_{i}^{i s} \leq \Gamma_{i}^{i r} \quad$ for $1 \leq s \leq n$,

If $\Gamma_{i}^{j r}$ and $A_{i}^{j}$ have opposite signs for $j \neq i$, then $\left|A_{i}^{j}\right| \leq\left|\Gamma_{i}^{j r}\right|$,

$\left|A_{i}^{j}+2 \Gamma_{i}^{j s}\right| \leq\left|A_{i}^{j}+2 \Gamma_{i}^{j r}\right|$ for $j \neq i, 1 \leq s \leq n$.

Proof: (i) Assumption 2 gives (25a), and (22a) for $r=0$ gives (25b). Condition (22b) gives $\left|A_{i}^{j}\right| \geq\left|A_{i}^{j}+2 \Gamma_{i}^{j s}\right|$ for $j \neq i$ and $1 \leq s \leq n$. If $\Gamma_{i}^{j s} \neq 0$, this can hold if and only if $A_{i}^{j}$ and $\Gamma_{i}^{j s}$ have opposite signs and $\left|A_{i}^{j}\right| \geq\left|\Gamma_{i}^{j s}\right|$, which yields (25c), (25d).

(ii) Equation (26a) follows from (22a) for $s=0$. Equation (26b) is Assumption 2 for $r$. Equation (26c) follows from (22a) and Assumption 2 for $s \geq 1$. Note that (26a) and (26b) imply that $A_{i}^{i}<0$, while (26b) and (26c) imply that $A_{i}^{i}+2 \Gamma_{i}^{i s}<0$ for all $1 \leq s \leq n$. Thus, (26a)-(26c) imply Assumption 2. Condition (22b) for $s=0$ gives $\left|A_{i}^{j}\right| \leq\left|A_{i}^{j}+2 \Gamma_{i}^{j r}\right|$. This is always true if either $A_{i}^{j}$ or $\Gamma_{i}^{j r}$ is 0 , or both have the same sign. If $A_{i}^{j}$ and $\Gamma_{i}^{j r}$ have opposite signs, then this holds if and only if $\left|A_{i}^{j}\right| \leq\left|\Gamma_{i}^{j r}\right|$. Equation (26e) follows from (22b) for $s \geq 1$.

\section{Examples of quadratic systems}

Example 4 (Explicit conditions for 2-species systems). Consider the 2-species system (11). Assume that $r(i)=0$ for $i=1,2$, so that conditions (25) hold for both species. If $\Gamma_{1}^{12} \neq 0$, for species- 1 , these conditions reduce to ${ }^{2}$ :

$$
\begin{aligned}
& A_{1}^{1}<0, \\
& \Gamma_{1}^{11}, \Gamma_{1}^{12}, \Gamma_{1}^{22} \leq 0, \\
& A_{1}^{2} \geq \max \left\{-\Gamma_{1}^{12},-\Gamma_{1}^{22}\right\} .
\end{aligned}
$$

Thus $\Gamma_{1}^{r s} \leq 0$ for all $r, s$, and $A_{1}^{2}$ is positive. In particular, these conditions imply that species-1 has a net negative direct effect on its own growth rate through the term $A_{1}^{1}$, but species- 2 has a net positive direct effect on species-1, through the term $A_{1}^{2}$, but no or negative effect through direct species-species interactions, through the terms $\Gamma_{1}^{r s} \leq 0$. Similar conditions apply for species-2.

Given these conditions, it remains to verify the single $\mathrm{H}-\mathrm{S}$ condition $\operatorname{det} \hat{F}>0$ :

$$
\operatorname{det} \hat{F}=\left(\begin{array}{cc}
\omega_{11}^{(0)} & -\left|\omega_{12}^{(0)}\right| \\
-\left|\omega_{21}^{(0)}\right| & \omega_{22}^{(0)}
\end{array}\right)=\left(\begin{array}{cc}
-A_{1}^{1} & -A_{1}^{2} \\
-A_{2}^{1} & -A_{2}^{2}
\end{array}\right),
$$

that is,

$$
A_{1}^{1} A_{2}^{2}>A_{2}^{1} A_{1}^{2} .
$$

\footnotetext{
${ }^{2}$ Condition (25b) implies that $\Gamma_{1}^{11}, \Gamma_{1}^{12} \leq 0$. If $\Gamma_{1}^{12} \neq 0$, then $\Gamma_{1}^{21}=\Gamma_{1}^{12}<0$ and (25c) with $i=s=1$ and $j=2$ implies that $\operatorname{sign} A_{1}^{2}=-\operatorname{sign} \Gamma_{1}^{21}=+1$. Hence, either $\Gamma_{1}^{22}=0$, or (25c) with $i=1$ and $j=s=2$ implies that $\operatorname{sign} \Gamma_{1}^{22}=-\operatorname{sign} A_{1}^{2}=-1$. That is, $\Gamma_{1}^{22} \leq 0$.
} 
The theory developed in previous sections implies that, subject to verification of the inward-pointing condition (Example 2), the above set of conditions on the coefficients of (11) is sufficient to ensure that there is a unique interior equilibrium that is globally asymptotically stable.

\section{Further discussion}

In terms of the notation of (6), and assuming $\gamma_{i}^{00}=0$, the system (11) is:

$$
\begin{aligned}
\dot{x}_{1}= & a_{1}^{0} x_{0}+a_{1}^{1} x_{1}+a_{1}^{2} x_{2}+2 \gamma_{1}^{01} x_{0} x_{1}+2 \gamma_{1}^{02} x_{0} x_{2}+\gamma_{1}^{11} x_{1}^{2} \\
& +2 \gamma_{1}^{12} x_{1} x_{2}+\gamma_{1}^{22} x_{2}^{2}, \\
\dot{x}_{2}= & a_{2}^{0} x_{0}+a_{2}^{1} x_{1}+a_{2}^{2} x_{2}+2 \gamma_{2}^{01} x_{0} x_{1}+2 \gamma_{2}^{02} x_{0} x_{2}+\gamma_{2}^{11} x_{1}^{2} \\
& +2 \gamma_{2}^{12} x_{1} x_{2}+\gamma_{2}^{22} x_{2}^{2} .
\end{aligned}
$$

Note from (9a) that $c_{i}=a_{i}^{0}$, and hence to obtain the inward-pointing conditions (12a), (12b), we require $a_{i}^{0}>0$ for $i=1,2$. Thus, there is positive external recruitment to available substrate for each species.

From (9c), $\Gamma_{i}^{j k}=\gamma_{i}^{j k}-\left(\gamma_{i}^{j 0}+\gamma_{i}^{0 k}\right)$, which is non-positive by (25b). Thus, $\gamma_{i}^{j 0}+\gamma_{i}^{0 k} \geq \gamma_{i}^{j k}$. This gives the relations:
(i) $2 \gamma_{1}^{01} \geq \gamma_{1}^{11}$,
(ii) $\gamma_{1}^{01}+\gamma_{1}^{02} \geq \gamma_{1}^{12}$,
(iii) $2 \gamma_{1}^{02} \geq \gamma_{1}^{22}$,
(i) $2 \gamma_{2}^{01} \geq \gamma_{2}^{11}$,
(ii) $\gamma_{2}^{01}+\gamma_{2}^{02} \geq \gamma_{2}^{12}$,
(iii) $2 \gamma_{2}^{02} \geq \gamma_{2}^{22}$.

We also require $A_{i}^{i}<0$, and from (9b) this implies:

$$
\text { (i) } 2 \gamma_{1}^{01}+a_{1}^{1}<a_{1}^{0}, \quad \text { (ii) } 2 \gamma_{2}^{02}+a_{2}^{2}<a_{2}^{0} \text {. }
$$

If we interpret $\rho_{i}=-a_{i}^{i}$ as the (positive) death rate of species $i$, then $a_{i}^{i}<0$. On the other hand, $\lambda_{i}=2 \gamma_{i}^{0 i}$ can be interpreted as the growth rate of species $i$ due to utilization of the substrate if it is positive, or as a density-dependent inhibition of recruitment and/or growth if it is negative (Section 2). In the latter case $\left(\lambda_{i} \leq 0\right),(31)$ always holds. In the former case $\left(\lambda_{i}>0\right),(31)$ can be interpreted as:

Growth rate - death rate $<$ recruitment rate.

This always holds, for example, if the death rate is greater than the growth rate, in which case the species cannot persist without external recruitment. We call systems satisfying (32) essentially recruitment limited.

Finally, (9b), (9c) together with (27c) yields:
(i) $a_{1}^{2}+\gamma_{1}^{02}+\gamma_{1}^{12}-\gamma_{1}^{01} \geq a_{1}^{0}$,
(ii) $a_{1}^{2}+\gamma_{1}^{22} \geq a_{1}^{0}$,
(i) $a_{2}^{1}+\gamma_{2}^{01}+\gamma_{2}^{12}-\gamma_{2}^{02} \geq a_{2}^{0}$,
(ii) $a_{2}^{1}+\gamma_{2}^{11} \geq a_{2}^{0}$. 
Since $a_{1}^{0}>0$, (33a) implies that there must be net positive effects of species-2 on the growth rate of species-1, acting either directly through $a_{1}^{2}$ or indirectly through pairwise interactions. In particular, if $a_{1}^{2}=0$, then (30a)(iii) and (33a)(ii) imply that $\gamma_{1}^{02}$ and $\gamma_{1}^{22}$ must be positive (in fact $2 \gamma_{1}^{02} \geq \gamma_{1}^{22} \geq a_{1}^{0}>0$ ). That is, species-2 has the effect of "preparing the ground" (rendering the resource environment more favourable) for the growth of species- 1 . However, this does not preclude the possibility that $\gamma_{1}^{12}$ is negative (though from (33a)(i) this requires $2 \gamma_{1}^{02}>2 a_{1}^{0}+\lambda_{1}$ ); i.e. direct interactions between the two species are detrimental to the growth rate of species-1. Analogous considerations apply to the effects of species- 1 on species- 2 .

Example 5 (Generalized Lotka-Volterra systems). Consider an $n$-species system of the form

$$
\dot{x}_{i}=a_{i}^{0} x_{0}+a_{i}^{i} x_{i}+2 \gamma_{i}^{0 i} x_{0} x_{i}+\sum_{j=1}^{n} \gamma_{i}^{i j} x_{i} x_{j} .
$$

The coefficients may be interpreted as: $a_{i}^{0}>0$ is the (positive) recruitment rate of species $i$ onto available substrate (represented by $x_{0}$ ) from the external environment; $\rho_{i}=-a_{i}^{i}>0$ is the mortality rate of species $i ; \lambda_{i}=2 \gamma_{i}^{0 i}$ is the utilization rate by established species $i$ (represented by $x_{i}$ ) of available substrate (represented by $x_{0}$ ) when this is positive, and is a density-dependent inhibition of recruitment and/or growth rate when it is negative. Finally, $\gamma_{i}^{i j}$ represents the effect (positive, negative or zero) on the growth rate of species $i$ resulting from direct interaction with species $j$. We refer to a system of the form (34) as a generalized Lotka-Volterra (L-V) system. Note that, when $x_{0}=0$, it reduces to a classical L-V system of the form defined by Eq. (1). However, the inward-pointing condition that we require implies that $x_{0}$ cannot remain equal to zero.

In particular, when $x_{i}=0, \dot{x}_{i}=a_{i}^{0} x_{0}$, so that the species $i$ population is re-established through recruitment from the external environment onto available substrate (unless there is no available substrate; i.e. unless $x_{0}=0$ ). Species $i$ is then in competition with every other species to recruit onto this substrate. If there is no available substrate (i.e. $x_{0}=0$ ), then $x_{1}+x_{2}+\cdots+x_{n}=1$, and $\dot{x}_{0}=-\dot{x}_{1}-\dot{x}_{2}-\cdots-\dot{x}_{n}$, which yields:

$$
\dot{x}_{0}=\sum_{i=1}^{n}\left\{-a_{i}^{i}-\sum_{j=1}^{n} \gamma_{i}^{i j} x_{j}\right\} x_{i} .
$$

To obtain the inward-pointing condition, we require this to be positive (so that substrate becomes instantaneously available); i.e.

$$
\sum_{j=1}^{n} \gamma_{i}^{i j} x_{j}<-a_{i}^{i} \quad \text { whenever } \sum_{j=1}^{n} x_{j}=1 .
$$

Recalling that $\rho_{i}=-a_{i}^{i}>0$, this condition holds if and only if

$$
\gamma_{i}^{i j}<\rho_{i} \quad \text { for all } 1 \leq i, j \leq n
$$


From $(9 b),(9 c)$, with $\gamma_{i}^{00}=0$, we have:

$$
\begin{aligned}
& A_{i}^{i}=\lambda_{i}-\rho_{i}-a_{i}^{0}, \\
& A_{i}^{j}=-a_{i}^{0} \quad \text { for } j \neq i, \\
& \Gamma_{i}^{i i}=\gamma_{i}^{i i}-\lambda_{i}, \\
& \Gamma_{i}^{i j}=\gamma_{i}^{i j}-\frac{1}{2} \lambda_{i} \quad \text { for } j \neq i, \\
& \Gamma_{i}^{j k}=0 \quad \text { for } i \notin\{j, k\} .
\end{aligned}
$$

The Assumption 2 conditions $A_{i}^{i}<0$ again imply that growth rate minus death rate is less than external recruitment rate, as in (32): i.e.

$$
\lambda_{i}-\rho_{i}<a_{i}^{0} .
$$

It is convenient to analyse this system in the case in which $\Omega=-D g$ has a uniformly column-dominant diagonal. As discussed in Section 4, explicit conditions implying the existence and global asymptotic stability of a unique equilibrium are determined by a function $r:\{1, \ldots, n\} \rightarrow\{0,1, \ldots, n\}$. We show in Appendix A that the explicit conditions (25) arising from the choice $r(i)=0$ are only possible for generalized L-V systems if $\Gamma_{i}^{i j}=0$ for all $j \neq i$. In this case, the set of indices $i$ for which $r(i)=0$ defines a semi-neutral subsystem, of the form (13), of the generalized L-V system.

Here we consider only functions $r:\{1, \ldots, n\} \rightarrow\{1, \ldots, n\}$, with associated explicit conditions of the form (26) (transposed for column dominance). The form of these conditions for a general function $r$ is complicated (see Appendix A). As an illustration, we consider only the simple case in which $r$ is the identity function $r(i)=i$. In this case, conditions (26) are as follows (see Appendix A) ${ }^{3}$ :

$$
\begin{array}{ll}
\text { (26a): } & \gamma_{i}^{i i} \geq \lambda_{i}, \\
\text { (26b): } & 2 \gamma_{i}^{i i}<\lambda_{i}+\rho_{i}+a_{i}^{0}, \\
\text { (26c): } & \lambda_{i} \leq 2\left(\gamma_{i}^{i i}-\gamma_{i}^{i j}\right), \quad j \neq i, \\
\text { (26e): } & \lambda_{j} \leq 2 \gamma_{j}^{i j} \leq 2 a_{j}^{0}+\lambda_{j}, \quad j \neq i .
\end{array}
$$

Clearly, (38c), (38d) imply that $0 \leq \gamma_{j}^{i j} \leq \gamma_{j}^{j j}$ for $j \neq i$ if $\lambda_{j} \geq 0$. Hence, the system (34) is cooperative in this case. However, if $\lambda_{j}<0$, the signs of $\gamma_{j}^{i j}$ and $\gamma_{j}^{j j}$ are undetermined by these conditions.

It now follows from (20) and (21) that $f_{i i}=\omega_{i i}^{(i)}$ and $f_{i j}=\left|\omega_{j i}^{(i)}\right|$ for $j \neq i$. Thus, $f_{i i}=-A_{i}^{i}-2 \Gamma_{i}^{i i}=a_{i}^{0}+\lambda_{i}+\rho_{i}-2 \gamma_{i}^{i i}$ and $f_{i j}=\left|A_{j}^{i}+2 \Gamma_{j}^{i i}\right|=\left|A_{j}^{i}\right|=a_{j}^{0}$ by (36b), (36e). Thus, the derived matrix $\hat{F}=\left(\hat{f}_{i j}\right)$ has entries $\hat{f}_{i i}=f_{i i}$, and $\hat{f}_{i j}=-f_{i j}=-a_{j}^{0}$. We conclude from Theorem 5 that, if the H-S conditions (17) hold for $\hat{F}$, the system (34) has a unique interior equilibrium $\bar{x}$ that is globally asymptotically stable. That is, we require

\footnotetext{
${ }^{3}$ In Appendix A we show that condition (26d) always holds for generalized L-V systems.
} 


$$
\hat{F}_{(k)}=\operatorname{det}\left(\begin{array}{cccc}
a_{1}^{0}+\lambda_{1}+\rho_{1}-2 \gamma_{1}^{11} & -a_{2}^{0} & \cdots & -a_{k}^{0} \\
-a_{1}^{0} & a_{2}^{0}+\lambda_{2}+\rho_{2}-2 \gamma_{2}^{22} & \cdots & -a_{k}^{0} \\
\vdots & \vdots & & \vdots \\
-a_{1}^{0} & -a_{2}^{0} & \cdots & a_{k}^{0}+\lambda_{k}+\rho_{k}-2 \gamma_{k}^{k k}
\end{array}\right)
$$

$>0$,

for $1 \leq k \leq n$. This follows from (38b) when $k=1$. To evaluate $\hat{F}_{(k)}$ for $k>1$, write

$$
R_{i}=1+\frac{\lambda_{i}+\rho_{i}-2 \gamma_{i}^{i i}}{a_{i}^{0}} .
$$

Condition (38b) implies that $R_{i}>0$. Then $\hat{F}_{(k)}$ can be written as

$$
\hat{F}_{(k)}=\left(\prod_{i=1}^{k} a_{i}^{0}\right) D\left\{R_{1}, \ldots, R_{k}\right\},
$$

where

$$
D\left\{R_{1}, \ldots, R_{k}\right\}=\operatorname{det}\left(\begin{array}{cccc}
R_{1} & -1 & \cdots & -1 \\
-1 & R_{2} & & \vdots \\
\vdots & \vdots & \ddots & -1 \\
-1 & -1 & \cdots & R_{k}
\end{array}\right) .
$$

Thus $\hat{F}_{(k)}>0$ if and only if $D\left\{R_{1}, \ldots, R_{k}\right\}>0$.

For example,

$$
D\left\{R_{1}, R_{2}\right\}=\left(1+R_{1}\right)\left(1+R_{2}\right)-\left(1+R_{1}\right)-\left(1+R_{2}\right)=R_{1} R_{2}-1,
$$

and

$$
\begin{aligned}
D & \left\{R_{1}, R_{2}, R_{3}\right\} \\
= & \left(1+R_{1}\right)\left(1+R_{2}\right)\left(1+R_{3}\right)-\left(1+R_{1}\right)\left(1+R_{2}\right)-\left(1+R_{2}\right)\left(1+R_{3}\right) \\
& \quad-\left(1+R_{1}\right)\left(1+R_{3}\right) \\
= & R_{1} R_{2} R_{3}-R_{1}-R_{2}-R_{3}-2 .
\end{aligned}
$$

These cases are illustrated in Fig. 2.

More generally, in the matrix in (40), subtract the $(i-1)$-th row from the $i$ th row for $1<i \leq k$ to obtain

$$
D\left\{R_{1}, \ldots, R_{k}\right\}=\operatorname{det}\left(\begin{array}{ccccc}
R_{1} & -1 & \cdots & -1 & -1 \\
-\left(1+R_{1}\right) & 1+R_{2} & \cdots & 0 & 0 \\
\vdots & \vdots & \ddots & \vdots & \vdots \\
0 & 0 & \ddots & 1+R_{k-1} & 0 \\
0 & 0 & \cdots & -\left(1+R_{k-1}\right) & 1+R_{k}
\end{array}\right) .
$$




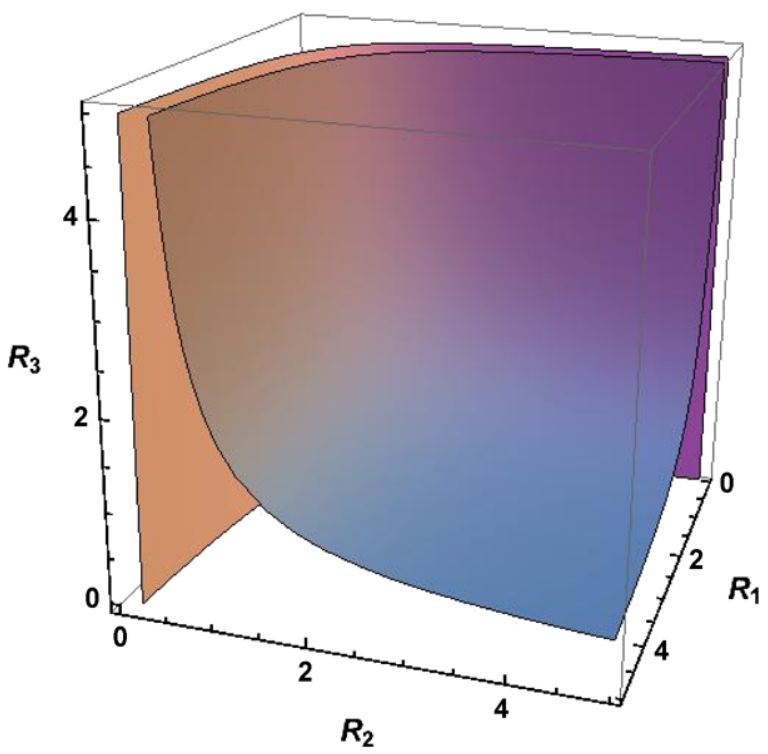

Fig. 2 The darker surface is $D\left\{R_{1}, R_{2}, R_{3}\right\}=R_{1} R_{2} R_{3}-R_{1}-R_{2}-R_{3}-2=0$ in the positive quadrant of $\left(R_{1}, R_{2}, R_{3}\right)$-space. The lighter surface is $D\left\{R_{1}, R_{2}\right\}=R_{1} R_{2}-1=0$. The condition $D\left\{R_{1}, R_{2}, R_{3}\right\}>0$ holds in the region lying above the dark grey surface. The condition $D\left\{R_{1}, R_{2}\right\}>0$ holds in the region above the light grey surface in the plane $R_{3}=0$

Now expand the determinant by the $k$ th column to obtain

$$
D\left\{R_{1}, \ldots, R_{k}\right\}=\left(1+R_{k}\right) D\left\{R_{1}, \ldots, R_{k-1}\right\}-\prod_{i=1}^{k-1}\left(1+R_{i}\right) .
$$

This shows that $D\left\{R_{1}, \ldots, R_{k}\right\}>0$ implies $D\left\{R_{1}, \ldots, R_{k-1}\right\}>0$. Hence, the H-S conditions reduce to the single condition $D\left\{R_{1}, \ldots, R_{n}\right\}>0$.

Clearly, $D\left\{R_{1}\right\}=R_{1}$, and by induction on $n \geq 2$ using (41), we obtain:

$$
D\left\{R_{1}, \ldots, R_{n}\right\}=\prod_{i=1}^{n}\left(1+R_{i}\right)-\sum_{i=1}^{n} \prod_{\substack{j=1 \\ j \neq i}}^{n}\left(1+R_{j}\right) \quad \text { for } n \geq 2 .
$$

It follows from (42) that the condition $D\left\{R_{1}, \ldots, R_{n}\right\}>0$ holds if and only if

$$
\sum_{i=1}^{n} \frac{1}{1+R_{i}}<1
$$

This result shows the existence of a large class of globally asymptotically stable ecosystems of generalized Lotka-Volterra type. However, it also shows that, the more species there are in the system (i.e. the larger $n$ is), the larger, on average, the coefficients $R_{i}$ must be to maintain the stability condition (43). 


\section{Discussion}

In this paper we have introduced and presented a preliminary analysis of a class of 'resource-bounded' model ecosystems, designed to model a fundamental aspect of interspecific competition: exploitation competition (Miller, 1967). This refers to competition arising from the joint exploitation by several species of a common limiting resource pool, which we refer to as the underlying substrate. This is in contrast to most analyses of classical Lotka-Volterra (L-V) systems, defined by Eq. (1), which focus on direct species-tospecies interactions, whether competitive or otherwise; i.e. on properties of the community matrix (e.g. MacArthur, 1970; May, 1973; Shigesada et al. 1984, 1989; reviewed in Hofbauer and Sigmund, 1998, Chap. 15).

The models we consider admit several interpretations-hence our use of the neutral term 'substrate' for the underlying resource. For example, the common resource could be space into, or over which sessile organisms can grow, such as assemblages of terrestrial plants, or marine benthic organisms such as corals (with their associated fish stocks), which recruit from planktonic larval pools that may be supplied from many non-local sources. A particular focus of recent research interest is the evolutionary ecology of microbial communities, whether organized as biofilms or host-adapted 'commensal' communities. Such communities are thought to exhibit a variety of complex interactions, both direct and indirect, many mediated by signalling molecules. A feature of these communities is their exposure to fluxes from the external environment of the host, their adaptation to particular micro-environments within the host, and their extreme resilience in the face of disturbance (Czárán et al., 2002; Horner-Devine et al., 2003; Rainey et al., 2005; Ley et al., 2006; IWA Task Group, 2006; Dethlefsen et al., 2007). Alternatively, the substrate could be interpreted in the sense of (bio-)chemistry, with the 'species' being varieties of molecule that react both with the substrate and each other to form a biochemical reaction system (Érdi and Tóth, 1989).

The results we have obtained concern conditions under which these model ecosystems admit a unique (internal) equilibrium that is globally asymptotically stable. However, the conditions we have found are rather general in character, and we have not focused here on applications to specific example systems that have particular biological characteristics. Nevertheless, an aspect of these globally stable systems we have emphasized is the important role of exogenous recruitment of constituent species onto substrate. Such recruitment guarantees that none of the constituent species can go extinct (though their equilibrium representation may be small), and, technically, is necessary for the associated vector field to be inward pointing on the boundary of state space (a simplex-see Section 2.2). This condition allows us to apply the powerful index theory and the Poincaré-Hopf Theorem (see Section 3.1).

The technical apparatus we have assembled allows us to construct suitable global Lyapunov functions. In particular, the starkest form of exploitation competition arises in what we refer to as 'neutral systems' (Section 3.2). These are systems in which the constituent species react only with the substrate and not directly with each other. If we also allow self-interference within each species (intra-specific competition), we call these systems 'semi-neutral'. By constructing an explicit Lyapunov function, we show that such systems are always globally stable whenever there is positive exogenous recruitment of each species. However, as exogenous recruitment of a species tends to zero, the species will become extinct through competitive exclusion. Versions of such neutral systems, which 
incorporate stochastic exogenous recruitment (and by implication, elimination) have been proposed as models that better account for the characteristics of real ecological communities than do traditional interaction-competition models (Hubbell, 2001). However, the role of interaction competition remains unresolved (e.g. Tilman, 2004, who also emphasizes the key role of exogenous recruitment). Our stability result for deterministic versions of these neutral and semi-neutral models is clearly relevant to this debate, in that it shows how stable ecosystems can arise from assemblages of species with only intrinsic properties.

For more general systems, which admit direct inter-species interactions, we generalize the result of Ikeda and Siljak (1980) for classical L-V systems, that if the community matrix has a dominant diagonal, then it is dissipative, and hence the system is globally stable when it admits a positive equilibrium point. Our generalization requires consideration of an extended form of diagonal dominance, which we call 'uniform diagonal dominance'. This property is used to construct suitable global Lyapunov functions (Theorems 2, 3). We consider in detail explicit conditions under which uniform diagonal dominance holds (Propositions 3 and 5) and unpack the implications for global stability (Theorems 4 and 5). In particular, these conditions are applied to quadratic systems (Section 6).

The explicit conditions for an $n$-species system derived in Proposition 5 are specified by a set-function $r:\{1,2, \ldots, n\} \rightarrow\{0,1,2, \ldots, n\}$. Each such specification determines a class of ecosystem models, and for each system in this class, an associated $n \times n$ matrix $\hat{F}$. The system is globally stable provided $\hat{F}$ is an $M$-matrix; i.e. the Hawkins-Simon conditions (17) are satisfied. The examples of quadratic systems we consider in Section 6 are defined by two simple choices of a set-function $r$ : two-species systems with $r(1)=r(2)=0$ (Example 4), and $n$-species generalized L-V systems with $r(i)=i$ (Example 5), though for the latter we detail the constraints arising from more general set-functions in Appendix A. In particular, the set of species $i$ for which $r(i)=0$ defines (with suitably modified parameters) a semi-neutral subsystem of the generalized L-V system. Thus, when $r$ is the identity function, the generalized L-V system has no such semi-neutral subsystems. For species in such a subsystem, competition is purely exploitative, via the substrate. However, species in this subsystem may have direct interactive effects on species outside it.

In addition to the positive exogenous recruitment rates, $a_{i}^{0}$, other key parameters that feature in our analyses are the net species death (or emigration) rates $\rho_{i}$, and the speciessubstrate interaction coefficients, $\lambda_{i}=2 \gamma_{i}^{i 0}$. We consider two possible interpretations for the latter:

a. If $\lambda_{i}>0$, it can be interpreted as the utilization rate of substrate by established representatives of species $i$.

b. If $\lambda_{i}<0$, it can be interpreted as a density-dependent inhibition of recruitment and/or substrate-utilization by established species $i$.

For example, in case a, if the substrate is space over which sessile organisms grow, then $\lambda_{i}$ is just the lateral growth rate of species $i$. In case $\mathrm{b}$, residents could inhibit each other's growth, or could render establishment of new recruits more difficult through resident advantage in gaining necessary resources. These interpretations have implications for the examples we consider in Section 6. Thus, for both Examples 4 and 5, in interpretation a $\left(\lambda_{i}>0\right)$, the explicit conditions imply that the ecosystem must be 'essentially recruitment limited', in the sense that growth rate - death rate $<$ recruitment rate. However, in interpretation b, this condition is automatically satisfied, and so does not constrain the system. 
These interpretations also have consequences for how the explicit conditions constrain the direct species-to-species interaction coefficients, $\gamma_{i}^{j k}$ (the effect on the growth rate of species $i$ due to direct interactions between species $j$ and species $k$ ). In particular, for the generalized L-V systems considered in Example 5, the coefficients $\gamma_{i}^{i j}$ cannot be negative under interpretation a, so that these systems are essentially cooperative. However, under interpretation $b$, there are no such limitations on the sign of these coefficients. Of course, mixed interpretations are possible, with $\lambda_{i}=\lambda_{i}^{a}-\lambda_{i}^{b}$, where $\lambda_{i}^{a}$ is a substrate utilization rate and $\lambda_{i}^{b}$ is a (positive) density-dependent inhibition rate. Which of these effects dominates, determines the sign of $\lambda_{i}$.

There is still much work to be done in understanding the systems considered in this paper. In particular, it will be instructive to map out in detail the ecological implications of the various possible explicit conditions arising from Proposition 5. Further, stochastic effects, particularly as they affect exogenous recruitment, are clearly fundamental, though our stability results imply that, once established, many multi-species communities will be extremely resilient in the face of disturbances. However, the main message of this paper is that there is a large and rich class of very robust (i.e. globally asymptotically stable) resource-bounded model ecosystems, based on exploitation competition of an underlying limiting resource, which should find application in modelling a variety of real-world domains.

\section{Acknowledgements}

We are grateful for discussions with Stephen Baigent. The presentation of the paper was significantly improved by the comments of two anonymous referees. R.M.S. was supported by the Centre for Mathematics and Physics in the Life Sciences and Experimental Biology (CoMPLEX); G.K. and T.F. were both supported by EPSRC studentships awarded by CoMPLEX.

\section{Appendix A}

\section{A.1 Semi-neutral systems}

Consider a semi-neutral system of the form (13) described in Section 3.2. Here we prove the assertion that $L(x)=\frac{1}{2} g(x) \cdot Q(x) g(x)$, with $Q(x)=\operatorname{diag}\left\{d_{1}\left(x_{1}\right), \ldots, d_{n}\left(x_{n}\right)\right\}$ and $d_{i}\left(x_{i}\right)=1 /\left(a_{i}^{0}+\lambda_{i} x_{i}\right)$ given by (14), defines a global Lyapunov function for the system (13). From (13) and (14):

$$
\frac{\partial \dot{x}_{i}}{\partial x_{j}}=-\left(a_{i}^{0}+\lambda_{i} x_{i}\right)-\delta_{i j}\left(\rho_{i}-\lambda_{i} x_{0}+2 \kappa_{i} x_{i}\right)=-\frac{1}{d_{i}\left(x_{i}\right)}-\delta_{i j} c_{i}(x),
$$

where $c_{i}(x)=\rho_{i}-\lambda_{i} x_{0}+2 \kappa_{i} x_{i}$. Note that $c_{i}(x)>0$, since we are assuming that $\rho_{i}>\lambda_{i}$ and $\kappa_{i} \geq 0$. It follows that

$$
-Q(x) D g(x)=E+\operatorname{diag}\left\{d_{1}\left(x_{1}\right) c_{1}(x), \ldots, d_{n}\left(x_{n}\right) c_{n}(x)\right\},
$$


where $E$ is the constant matrix all of whose entries are 1. Clearly, the diagonal matrix is positive definite. Also, for any $u \in \mathbf{R}^{n}$, we have $E u=(e \cdot u) e$, where $e \in \mathbf{R}^{n}$ is the vector all of whose entries are 1 . Thus $u \cdot E u=(e \cdot u)^{2} \geq 0$. It follows that $Q(x) D g(x)$ is negative definite for each $x \in \Delta$, and hence that the eigenvalues of $D g(x)$ all have negative real parts. The Index Theorem (Section 3.1) then guarantees the existence of a unique equilibrium $\bar{x} \in \operatorname{int} \Delta$.

Now consider $\dot{Q}(x)=\operatorname{diag}\left\{\dot{d}_{1}\left(x_{1}\right), \ldots, \dot{d}_{n}\left(x_{n}\right)\right\}$. We have:

$$
\dot{d}_{i}\left(x_{i}\right)=-\frac{\lambda_{i} \dot{x}_{i}}{\left(a_{i}^{0}+\lambda_{i} x_{i}\right)^{2}}=-d_{i}(x) \frac{\lambda_{i} \dot{x}_{i}}{a_{i}^{0}+\lambda_{i} x_{i}},
$$

and from (13):

$$
\begin{aligned}
\frac{\lambda_{i} \dot{x}_{i}}{a_{i}^{0}+\lambda_{i} x_{i}} & =\lambda_{i} x_{0}-\left(\frac{\lambda_{i} x_{i}}{a_{i}^{0}+\lambda_{i} x_{i}}\right)\left(\rho_{i}+\kappa_{i} x_{i}\right) \\
& =\lambda_{i} x_{0}-\left(1-\frac{a_{i}^{0}}{a_{i}^{0}+\lambda_{i} x_{i}}\right)\left(\rho_{i}+\kappa_{i} x_{i}\right) \\
& =\left(\frac{a_{i}^{0}}{a_{i}^{0}+\lambda_{i} x_{i}}\right)\left(\rho_{i}+\kappa_{i} x_{i}\right)+\kappa_{i} x_{i}-\left(\rho_{i}-\lambda_{i} x_{0}+2 \kappa_{i} x_{i}\right) \\
& =d_{i}\left(x_{i}\right) a_{i}^{0}\left(\rho_{i}+\kappa_{i} x_{i}\right)+\kappa_{i} x_{i}-c_{i}(x) .
\end{aligned}
$$

Thus:

$$
\dot{d}_{i}\left(x_{i}\right)=d_{i}\left(x_{i}\right) c_{i}(x)-d_{i}\left(x_{i}\right) h_{i}(x),
$$

where $h_{i}(x)=\kappa_{i} x_{i}+d_{i}\left(x_{i}\right) a_{i}^{0}\left(\rho_{i}+\kappa_{i} x_{i}\right)$, which is clearly positive. Hence:

$$
\begin{aligned}
\dot{Q}(x)= & \operatorname{diag}\left\{d_{1}\left(x_{1}\right) c_{1}(x), \ldots, d_{n}\left(x_{n}\right) c_{n}(x)\right\} \\
& -\operatorname{diag}\left\{d_{1}\left(x_{1}\right) h_{1}(x), \ldots, d_{n}\left(x_{n}\right) h_{n}(x)\right\} .
\end{aligned}
$$

We now obtain:

$$
\begin{aligned}
-\dot{L}(x)= & g(x) \cdot\left[-Q(x) D g(x)-\frac{1}{2} \dot{Q}(x)\right] g(x) \\
= & g(x) \cdot\left[E+\operatorname{diag}\left\{d_{1}\left(x_{1}\right) c_{1}(x), \ldots, d_{n}\left(x_{n}\right) c_{n}(x)\right\}\right. \\
& -\frac{1}{2} \operatorname{diag}\left\{d_{1}\left(x_{1}\right) c_{1}(x), \ldots, d_{n}\left(x_{n}\right) c_{n}(x)\right\} \\
& \left.+\frac{1}{2} \operatorname{diag}\left\{d_{1}\left(x_{1}\right) h_{1}(x), \ldots, d_{n}\left(x_{n}\right) h_{n}(x)\right\}\right] g(x) \\
= & g(x) \cdot\left[E+\frac{1}{2} \operatorname{diag}\left\{d_{1}\left(x_{1}\right) c_{1}(x), \ldots, d_{n}\left(x_{n}\right) c_{n}(x)\right\}\right. \\
& \left.+\frac{1}{2} \operatorname{diag}\left\{d_{1}\left(x_{1}\right) h_{1}(x), \ldots, d_{n}\left(x_{n}\right) h_{n}(x)\right\}\right] g(x) .
\end{aligned}
$$


Clearly the diagonal matrices in this expression are positive definite. Also, as noted above, $u \cdot E u=(e \cdot u)^{2} \geq 0$. It follows that $\dot{L}(x)<0$ for all $x \neq \bar{x}$, and hence that $L(x)$ is a global Lyapunov function for this system.

In fact, using the formulae (15), we can derive the unique equilibrium for semi-neutral systems explicitly. Thus, adding the formulae (15) for $1 \leq i \leq n$ and using (5) gives the relation $H\left(\bar{x}_{0}\right)=1$, where

$$
H\left(x_{0}\right)=x_{0}+\sum_{i=1}^{n} \frac{1}{2 \kappa_{i}}\left\{-\left(\rho_{i}-\lambda_{i} x_{0}\right)+\sqrt{\left(\rho_{i}-\lambda_{i} x_{0}\right)^{2}+4 \kappa_{i} a_{i}^{0} x_{0}}\right\} .
$$

Clearly $H(0)=0$ and $H(1)>1$. Further, it is easily shown that $H\left(x_{0}\right)$ is a monotonically increasing function of $x_{0}$, and hence that there is a unique $\bar{x}_{0} \in(0,1)$ satisfying $H\left(\bar{x}_{0}\right)=1$. This provides an elementary proof of the uniqueness of equilibrium for semineutral systems.

\section{A.2 Proof of Theorem 2}

We adapt an argument originally due to Araki and Kondo (1972). Let $c$ and $d$ be positive vectors such that $\hat{\Omega} d>0$ and $\hat{\Omega}^{T} c>0$ (for brevity, the argument $x$ is temporarily suppressed). Let $C=\operatorname{diag}\left\{c_{1}, \ldots, c_{n}\right\}, D=\operatorname{diag}\left\{d_{1}, \ldots, d_{n}\right\}$ and $Q=C D^{-1}=$ $\operatorname{diag}\left\{q_{1}, \ldots, q_{n}\right\}$, where $q_{i}=c_{i} / d_{i}$. Note that $Q d=c$. Thus, $\left(Q \hat{\Omega}+\hat{\Omega}^{T} Q\right) d=Q \hat{\Omega} d+$ $\hat{\Omega}^{T} c>0$, since $Q$ is a positive diagonal matrix and hence $Q x>0$ for any positive vector $x$.

Now let $\Pi=Q \Omega+\Omega^{T} Q$. Then $\pi_{i j}=q_{i} \omega_{i j}+q_{j} \omega_{j i}$. Thus, $\hat{\pi}_{i i}=\left|\pi_{i i}\right|=2 q_{i}\left|\omega_{i i}\right|=$ $\left(Q \hat{\Omega}+\hat{\Omega}^{T} Q\right)_{i i}$, and for $j \neq i, \hat{\pi}_{i j}=-\left|\pi_{i j}\right| \geq-q_{i}\left|\omega_{i j}\right|-q_{j}\left|\omega_{j i}\right|=q_{i} \hat{\omega}_{i j}+q_{j} \hat{\omega}_{j i}=$ $\left(Q \hat{\Omega}+\hat{\Omega}^{T} Q\right)_{i j}$. It follows that

$$
(\hat{\Pi} d)_{i}=\sum_{j} \hat{\pi}_{i j} d_{j} \geq \sum_{j}\left(Q \hat{\Omega}+\hat{\Omega}^{T} Q\right)_{i j} d_{j}=\left[\left(Q \hat{\Omega}+\hat{\Omega}^{T} Q\right) d\right]_{i}>0,
$$

for each $i$, since $\left(Q \hat{\Omega}+\hat{\Omega}^{T} Q\right) d>0$. Reinstating the argument $x$, this shows that $\hat{\Pi}(x) d>0$ for all $x \in \Delta$ and hence that $\Pi(x)$ has a uniformly row-dominant diagonal. Since $\Pi(x)$ is symmetric and has positive diagonal entries, it is positive definite by Proposition 1. That is, $Q \Omega(x)$ is positive definite for all $x \in \Delta$. The result now follows from Theorem 1.

\section{A.3 Proof of Theorem 3}

By Assumption 2, $\Omega(x)$ has positive diagonal entries. Thus, if $\Omega(x)$ has a uniformly dominant diagonal (either row or column), then, by Proposition 1, the eigenvalues of $\Omega(x)$ all have positive real parts. Since $D g(x)=-\Omega(x)$, the eigenvalues of $D g(x)$ all have negative real parts, and hence that sign det $D g(x)=(-1)^{n}$ for all $x \in \Delta$. By the Index Theorem (Section 3.1), this implies that $\dot{x}=g(x)$ has exactly one equilibrium $\bar{x} \in$ int $\Delta$.

Let $v \in \mathbf{R}^{n}$ be a fixed vector, and suppose $x \in \Delta$ with $x \neq \bar{x}$. Since $\Delta$ is convex, $\theta x+(1-\theta) \bar{x} \in \Delta$ for $0 \leq \theta \leq 1$. Define a scalar function $\varphi_{v}:[0,1] \rightarrow \mathbf{R}$ by $\varphi_{v}(\theta)=$ $v \cdot g(\theta x+(1-\theta) \bar{x})$. Then

$$
\varphi_{v}^{\prime}(\theta)=v \cdot D g(\theta x+(1-\theta) \bar{x})(x-\bar{x}) .
$$


By the Mean Value Theorem, there is a $\theta \in(0,1)$ such that $\varphi_{v}(1)-\varphi_{v}(0)=\varphi_{v}^{\prime}(\theta)$. That is,

$$
v \cdot(g(x)-g(\bar{x}))=v \cdot D g(\theta x+(1-\theta) \bar{x})(x-\bar{x}) .
$$

Now define a function

$$
L_{v}(x)=v \cdot(x-\bar{x})
$$

Then, since $\dot{x}=g(x)$, it follows from (A.1) that

$$
\dot{L}_{v}(x)=v \cdot g(x)=v \cdot(g(x)-g(\bar{x}))=v \cdot D g(\tilde{x})(x-\bar{x}),
$$

where $\tilde{x}=\theta x+(1-\theta) \bar{x}$.

We consider two cases.

Case 1: First suppose that $\Omega=-D g$ has a uniformly column-dominant diagonal. Take $v$ to be the vector defined by $v_{i}=d_{i} \operatorname{sign}\left(x_{i}-\bar{x}_{i}\right)$. Then from (A.2), $L_{v}(x)=$ $\sum_{i=1}^{n} d_{i}\left|x_{i}-\bar{x}_{i}\right|$. Clearly, $L_{v}(x) \geq 0$ with equality if and only if $x=\bar{x}$. Also, from (A.3):

$$
\begin{aligned}
\dot{L}_{v}(x) & =-v \cdot \Omega(\tilde{x})(x-\bar{x})=-\sum_{i, j} v_{i} \omega_{i j}(\tilde{x})\left(x_{j}-\bar{x}_{j}\right) \\
& =-\sum_{i, j} d_{i} \operatorname{sign}\left(x_{i}-\bar{x}_{i}\right) \omega_{i j}(\tilde{x})\left(x_{j}-\bar{x}_{j}\right) \\
& \left.=-\sum_{i, j} d_{j} \operatorname{sign}\left(x_{j}-\bar{x}_{j}\right) \omega_{j i}(\tilde{x})\left(x_{i}-\bar{x}_{i}\right) \quad \text { (interchanging } i \text { and } j\right) \\
& =-\sum_{i=1}^{n}\left(x_{i}-\bar{x}_{i}\right)\left\{d_{i} \omega_{i i}(\tilde{x}) \operatorname{sign}\left(x_{i}-\bar{x}_{i}\right)+\sum_{j \neq i} d_{j} \omega_{j i}(\tilde{x}) \operatorname{sign}\left(x_{j}-\bar{x}_{j}\right)\right\} \\
& =-\sum_{i=1}^{n}\left|\left(x_{i}-\bar{x}_{i}\right)\right|\left\{d_{i} \omega_{i i}(\tilde{x})+\operatorname{sign}\left(x_{i}-\bar{x}_{i}\right) \sum_{j \neq i} d_{j} \omega_{j i}(\tilde{x}) \operatorname{sign}\left(x_{j}-\bar{x}_{j}\right)\right\} \\
& \leq-\sum_{i=1}^{n}\left|\left(x_{i}-\bar{x}_{i}\right)\right| d_{i} \omega_{i i}(\tilde{x})+\sum_{i=1}^{n}\left|\left(x_{i}-\bar{x}_{i}\right)\right| \sum_{j \neq i} d_{j}\left|\omega_{j i}(\tilde{x})\right| \\
& =-\sum_{i=1}^{n}\left|\left(x_{i}-\bar{x}_{i}\right)\right|\left\{d_{i} \omega_{i i}(\tilde{x})-\sum_{j \neq i} d_{j}\left|\omega_{j i}(\tilde{x})\right|\right\}<0,
\end{aligned}
$$

for $x \neq \bar{x}$, since $\Omega$ has a uniformly column-dominant diagonal. This shows that $L_{v}(x)$ is a global Lyapunov function on $\Delta$, and hence that $\bar{x}$ is globally asymptotically stable.

Case 2: Now suppose that $\Omega=-D g$ has a uniformly row-dominant diagonal. Let $i^{*}$ be an index for which

$$
\max _{i}\left\{\frac{\left|x_{i}-\bar{x}_{i}\right|}{d_{i}}\right\}=\frac{\left|x_{i^{*}}-\bar{x}_{i^{*}}\right|}{d_{i^{*}}},
$$


and in (A.2) take $v_{i}=0$ for $i \neq i^{*}$ and $v_{i^{*}}=\operatorname{sign}\left(x_{i^{*}}-\bar{x}_{i^{*}}\right) / d_{i^{*}}$. Then,

$$
L_{v}(x)=\frac{\left|x_{i^{*}}-\bar{x}_{i^{*}}\right|}{d_{i^{*}}} .
$$

Clearly, $L_{v}(x) \geq 0$ with equality if and only if $x=\bar{x}$. Also, from (A.3):

$$
\begin{aligned}
\dot{L}_{v}(x) & =-\frac{1}{d_{i^{*}}} \sum_{j} \operatorname{sign}\left(x_{i^{*}}-\bar{x}_{i^{*}}\right) \omega_{i^{*} j}(\tilde{x})\left(x_{j}-\bar{x}_{j}\right) \\
& =-\frac{1}{d_{i^{*}}}\left\{\left|x_{i^{*}}-\bar{x}_{i^{*}}\right| \omega_{i^{*} i^{*}}(\tilde{x})+\operatorname{sign}\left(x_{i^{*}}-\bar{x}_{i^{*}}\right) \sum_{j \neq i^{*}} \omega_{i^{*} j}(\tilde{x})\left(x_{j}-\bar{x}_{j}\right)\right\} \\
& \leq-\frac{1}{d_{i^{*}}}\left\{L_{v}(x) d_{i^{*}} \omega_{i^{*} i^{*}}(\tilde{x})-\sum_{j \neq i^{*}} d_{j}\left|\omega_{i^{*} j}(\tilde{x})\right| \frac{\left|x_{j}-\bar{x}_{j}\right|}{d_{j}}\right\} \\
& \leq-\frac{1}{d_{i^{*}}} L_{v}(x)\left\{d_{i^{*}} \omega_{i^{*} i^{*}}(\tilde{x})-\sum_{j \neq i^{*}} d_{j}\left|\omega_{i^{*} j}(\tilde{x})\right|\right\}<0
\end{aligned}
$$

for $x \neq \bar{x}$, since $\Omega$ has a uniformly row-dominant diagonal. This shows that $L_{v}(x)$ is a global Lyapunov function on $\Delta$, and hence that $\bar{x}$ is globally asymptotically stable.

\section{A.4 Generalized L-V systems}

Consider a function $r:\{1, \ldots, n\} \rightarrow\{0,1, \ldots, n\}$ defining explicit conditions for a generalized L-V system of the form (34), as discussed in Section 6.

First consider indices $i$ for which $r(i)=0$. If such an $i$ exists, then the corresponding explicit conditions are (25). For $s=j \neq i,(25 \mathrm{~b})$ and (36d) imply that $\Gamma_{j}^{j i}=\gamma_{j}^{j i}-$ $\frac{1}{2} \lambda_{j} \leq 0$. That is, $2 \gamma_{j}^{j i} \leq \lambda_{j}$. On the other hand, if $\Gamma_{j}^{j i} \neq 0$, then (25c) and (36b) with $s=i \neq j$ imply that $\operatorname{sign} \Gamma_{j}^{i j}=-\operatorname{sign} A_{j}^{i}=+1$, and hence $2 \gamma_{j}^{j i}>\lambda_{j}$. This is a contradiction. The only other possibility is $\Gamma_{j}^{j i}=0$ for all $i \neq j$. That is, $2 \gamma_{j}^{j i}=\lambda_{j}$.

If $i$ is an index for which $r(i)=0$, then substituting $\gamma_{i}^{i j}=\frac{1}{2} \lambda_{i}$ for $j \neq i$ and using $\sum_{j \neq i} x_{j}=1-x_{0}-x_{i}$, shows that Eq. (34) reduces to the form:

$$
\dot{x}_{i}=a_{i}^{0} x_{0}-\left(\rho_{i}-\frac{1}{2} \lambda_{i}\right) x_{i}+\frac{1}{2} \lambda_{i} x_{0} x_{i}-\left(\frac{1}{2} \lambda_{i}-\gamma_{i}^{i i}\right) x_{i}^{2},
$$

where $\rho_{i}=-a_{i}^{i}$ and $\lambda_{i}=2 \gamma_{i}^{i 0}$. This has the form of a semi-neutral system (13):

$$
\dot{x}_{i}=a_{i}^{0} x_{0}-\tilde{\rho}_{i} x_{i}+\tilde{\lambda}_{i} x_{0} x_{i}-\tilde{\kappa}_{i} x_{i}^{2},
$$

where $\tilde{\rho}_{i}=\rho_{i}-\frac{1}{2} \lambda_{i}, \tilde{\lambda}_{i}=\frac{1}{2} \lambda_{i}$ and $\tilde{\kappa}_{i}=\frac{1}{2} \lambda_{i}-\gamma_{i}^{i i}$. Note that (35) implies that $\rho_{i}>\gamma_{i}^{i j}=$ $\frac{1}{2} \lambda_{i}$ for any $j \neq i$, and hence $\tilde{\rho}_{i}>0$. Also, (25b) implies that $\Gamma_{i}^{i i} \leq 0$, and hence, from (36c), $\lambda_{i}-\gamma_{i}^{i i} \geq 0$. This gives $\tilde{\lambda}_{i}+\tilde{\kappa}_{i} \geq 0$. Note also that (25a) and (36a) imply that $\lambda_{i}-\rho_{i}<a_{i}^{0}$, and hence that $\tilde{\lambda}_{i}-\tilde{\rho}_{i}<a_{i}^{0}$. That is, the derived system (A.4) is essentially recruitment limited in the sense of (32). 
We have shown that the set of indices $i$ for which $r(i)=0$ and the explicit conditions (25) hold, determines a semi-neutral subsystem of the generalized L-V system.

Now consider indices $i$ for which $r(i) \neq 0$. The relevant explicit conditions are (26). We describe these conditions as they apply to generalized L-V systems below.

Condition (26a): $\Gamma_{i}^{i r(i)} \geq 0$.

$$
\begin{aligned}
& \text { If } r(i)=i,(36 \mathrm{c}) \quad \Rightarrow \quad \gamma_{i}^{i i} \geq \lambda_{i} . \\
& \text { If } r(i) \neq i,(36 \mathrm{~d}) \quad \Rightarrow \quad \gamma_{i}^{i r(i)} \geq \frac{1}{2} \lambda_{i} .
\end{aligned}
$$

Condition (26b): $A_{i}^{i}+2 \Gamma_{i}^{i r(i)}<0$.

$$
\begin{aligned}
& \text { If } r(i)=i,(36 \mathrm{a}),(36 \mathrm{c}) \quad \Rightarrow \quad 2 \gamma_{i}^{i i}<\lambda_{i}+\rho_{i}+a_{i}^{0} . \\
& \text { If } r(i) \neq i,(36 \mathrm{a}),(36 \mathrm{~d}) \quad \Rightarrow \quad 2 \gamma_{i}^{i r(i)}<\rho_{i}+a_{i}^{0} .
\end{aligned}
$$

Condition (26c): $\Gamma_{i}^{i s} \leq \Gamma_{i}^{i r(i)}$ for $1 \leq s \leq n$.

If $s=r(i)$, the condition holds trivially.

If $s=i \neq r(i)$, then the condition is $\Gamma_{i}^{i i} \leq \Gamma_{i}^{i r(i)}$. This reduces to $\gamma_{i}^{i i}-\lambda_{i} \leq \gamma_{i}^{i r(i)}-$ $\frac{1}{2} \lambda_{i}$, which gives:

$$
2\left(\gamma_{i}^{i i}-\gamma_{i}^{i r(i)}\right) \leq \lambda_{i}, \quad r(i) \neq i
$$

If $s=j \neq i, r(i)$, the condition is $\Gamma_{i}^{i j} \leq \Gamma_{i}^{i r(i)}$. That is, $\gamma_{i}^{i j}-\frac{1}{2} \lambda_{i} \leq \gamma_{i}^{i i}-\lambda_{i}$ if $r(i)=i$, and $\gamma_{i}^{i j}-\frac{1}{2} \lambda_{i} \leq \gamma_{i}^{i r(i)}-\frac{1}{2} \lambda_{i}$ if $r(i) \neq i$. Thus:

$$
\begin{aligned}
& \lambda_{i} \leq 2\left(\gamma_{i}^{i i}-\gamma_{i}^{i j}\right), \quad r(i)=i \neq j, \\
& \gamma_{i}^{i j} \leq \gamma_{i}^{i r(i)}, \quad r(i) \neq i \neq j \neq r(i) .
\end{aligned}
$$

Condition (26e): $\left|A_{j}^{i}+2 \Gamma_{j}^{i s}\right| \leq\left|A_{j}^{i}+2 \Gamma_{j}^{i r(i)}\right|$ for $j \neq i, s \geq 1$.

If $j \neq i, r(i)$ this reduces to $\left|A_{j}^{i}+2 \Gamma_{j}^{i s}\right| \leq\left|A_{j}^{i}\right|$ by (36e). If $s \neq j$ then $\Gamma_{j}^{i s}=0$ again by (36e), and the condition holds trivially. If $s=j$, the condition is: $\mid-a_{j}^{0}+$ $2 \gamma_{j}^{i j}-\lambda_{j} \mid \leq a_{j}^{0}$. This holds if and only if:

$$
\lambda_{j} \leq 2 \gamma_{j}^{i j} \leq \lambda_{j}+2 a_{j}^{0}, \quad j \neq i, r(i) .
$$

If $j=r(i) \neq i$, then (26e) reduces to $\left|A_{r(i)}^{i}+2 \Gamma_{r(i)}^{i s}\right| \leq\left|A_{r(i)}^{i}+2 \Gamma_{r(i)}^{i r(i)}\right|$. If $s=r(i)$, this holds trivially. If $s \neq r(i)$, the condition reduces to $\left|A_{r(i)}^{i}\right| \leq\left|A_{r(i)}^{i}+2 \Gamma_{r(i)}^{i r(i)}\right|$ by (36e). That is, $a_{r(i)}^{0} \leq\left|-a_{r(i)}^{0}+2 \gamma_{r(i)}^{i r(i)}-\lambda_{r(i)}\right|$, which gives

$$
\begin{array}{ll}
\text { Either: } & 2 \gamma_{r(i)}^{r(i) i} \leq \lambda_{r(i)}, \\
\text { Or: } & 2 \gamma_{r(i)}^{r(i) i} \geq \lambda_{r(i)}+2 a_{r(i)}^{0} .
\end{array}
$$


Condition (26d): If $\Gamma_{j}^{i r(i)}$ and $A_{j}^{i}$ have opposite signs for $j \neq i$, then $\left|A_{j}^{i}\right| \leq\left|\Gamma_{j}^{i r(i)}\right|$. Sign $A_{j}^{i}=-1$ by (36b), and so this condition is non-empty only if $\Gamma_{j}^{i r(i)}>0$.

If $j \neq i, r(i)$, then $\Gamma_{j}^{i r(i)}=0$ and the condition is empty.

If $j=r(i) \neq i$, then either $2 \Gamma_{j}^{i r(i)}=2 \Gamma_{j}^{i j}=2 \gamma_{j}^{i j}-\lambda_{j} \leq 0$ if (A.13) holds, in which case the condition is empty, or $2 \Gamma_{j}^{i r(i)}=2 \Gamma_{j}^{i j}=2 \gamma_{j}^{i j}-\lambda_{j} \geq 2 a_{j}^{0}>0$ if (A.14) holds, in which case the condition holds trivially since $\left|A_{j}^{i}\right|=a_{j}^{0}$.

Hence condition (26d) always holds for generalized L-V systems.

Open Access This article is distributed under the terms of the Creative Commons Attribution Noncommercial License which permits any noncommercial use, distribution, and reproduction in any medium, provided the original author(s) and source are credited.

\section{References}

Abrams, P.A., 1987. Alternative models of character displacement and niche shift. 2. Displacement when there is competition for a single resource. Am. Nat. 130, 271-282.

Araki, M., Kondo, B., 1972. Stability and transient behaviour of composite nonlinear systems. IEEE Trans. Automat. Control 17, 537-541.

Begon, M., Harper, J.L., Townsend, C.R., 1996. Ecology: Individuals, Populations and Communities. Blackwell Scientific, Oxford.

Bruno, J.F., Stachowicz, J.J., Bertness, M.D., 2003. Inclusion of facilitation into ecological theory. Trends Ecol. Evol. 18, 119-125.

Case, T.J., Casten, R.G., 1979. Global stability and multiple domains of attraction in ecological systems. Am. Nat. 113, 705-714.

Czárán, T.L., Hoekstra, R.F., Pagie, L., 2002. Chemical warfare between microbes promotes biodiversity. Proc. Natl. Acad. Soc. USA 99, 786-790.

Day, T., Young, K.A., 2004. Competitive and facilitative evolutionary diversification. BioScience 54, 101109.

Dethlefsen, L., McFall-Ngai, M., Relman, D.A., 2007. An ecological and evolutionary perspective on human-microbe mutualism and disease. Nature 449, 811-818.

Elton, C., 1958. The Ecology of Invasion by Animals and Plants. Methuen, London.

Érdi, P., Tóth, J., 1989. Mathematical Models of Chemical Reactions. Nonlinear Science: Theory and Applications. Manchester University Press, Manchester.

Gilpin, M.E., 1975. Stability of feasible predator-prey systems. Nature 254, 137-139.

Goh, B.S., Jennings, L.S., 1977. Feasibility and stability in randomly assembled Lotka-Volterra models. Ecol. Mod. 3, 63-71.

Harrison, G.W., 1979. Global stability of food chains. Am. Nat. 114, 455-457.

Hofbauer, J., Sigmund, K., 1998. Evolutionary Games and Population Dynamics. Cambridge University Press, Cambridge.

Horner-Devine, M.C., Carney, K.M., Bohannan, B.J.M., 2003. An ecological perspective on bacterial biodiversity. Proc. R. Soc. Lond. B 271, 113-122.

Hubbell, S.P., 2001. The Unified Neutral Theory of Biodiversity and Biogeography. Monographs in Population Biology. Princeton University Press, Princeton.

Ikeda, M., Siljak, D.D., 1980. Lotka-Volterra equations: decomposition, stability and structure. J. Math. Biol. 9, 65-83.

IWA Task Group on Biofilm Modeling, 2006. Mathematical Modeling of Biofilms, Scientific and Technical Report No. 18, IWA Publishing.

Kondoh, M., 2003. Foraging adaptation and the relationship between food-web complexity and stability. Science 299, 1388-1391.

Ley, R.E., Petersen, D.A., Gordon, J.I., 2006. Ecological and evolutionary forces shaping microbial diversity in the human intestine. Cell 124, 837-848.

Logofet, D.O., 1993. Matrices and Graphs: Stability Problems in Mathematical Ecology. CRC Press, Boca Raton. 
Loreau, M., Naeem, S., Inchausti, P., 2002. Biodiversity and Ecosystem Functioning. Oxford University Press, Oxford.

MacArthur, R.H., 1955. Fluctuations of animal populations, and a measure of community stability. Ecology $36,522-526$.

MacArthur, R.H., Levins, R., 1967. The limiting similarity, convergence, and divergence of coexisting species. Am. Nat. 101, 377-385.

MacArthur, R.H., 1970. Species packing and competitive equilibria for many species. Theor. Popul. Biol. $1,1-11$

May, R.M., 1972. Will a large complex ecosystem be stable? Nature 238, 413-414.

May, R.M., 1973. Stability and Complexity in Model Ecosystems. Monographs in Population Biology, vol. 6. Princeton University Press, Princeton.

Miller, R.S., 1967. Pattern and process in competition. Adv. Ecol. Res 4, 1-74.

Milnor, J.W., 1965. Topology from the Differentiable Viewpoint. University of Virginia Press, Charlottesville.

Rainey, P.B., Brockhurst, M.A., Buckling, A., Hodgson, D.J., Kassen, R., 2005. The use of model Pseudomonas fluorescens populations to study the causes and consequences of microbial diversity. In: Bardgett, R., Hopkins, D., Usher, M. (Eds.), Soil Biodiversity and Ecosystem Function, Cambridge University Press, Cambridge.

Shigesada, N., Kawasaki, K., Teramoto, E., 1984. The effect of interference competition on stability, structure and invasion of a multi-species system. J. Math. Biol. 21, 97-113.

Shigesada, N., Kawasaki, K., Teramoto, E., 1989. Direct and indirect effects of invasions of predators on a multiple-species community. Theor. Popul. Biol. 36, 311-338.

Siljak, D.D., 1978. Large Scale Dynamical Systems: Stability and Structures. North-Holland, Amsterdam.

Takayama, A., 1985. Mathematical Economics, 2nd edn. Cambridge University Press, Cambridge.

Tilman, D., 2004. Niche trade-offs, neutrality, and community structure: a stochastic theory of resource competition, invasion, and community assembly. Proc. Natl. Acad. Soc. USA 101, 10845-11176.

Volterra, V., 1931. Leçons sur la théorie mathématique de la lutte pour la vie. Gauthier-Villars, Paris.

Weiher, E., Keddy, P., 1999. Ecological Assembly Rules: Perspectives, Advances, and Retreats. Cambridge University Press, Cambridge. 